CTP TAMU-1/94

Göteborg ITP 94-3

Imperial/TP/93-94/13

hep-th/9401007

January 1994

\title{
The Multivalued Free-Field Maps of Liouville and Toda Gravities*
}

\author{
A. Anderson ${ }^{1}$, B.E.W. Nilsson ${ }^{2}$, C.N. Pope $^{3 \dagger}$ and K.S. Stelle ${ }^{1}$ \\ 1 The Blackett Laboratory, Imperial College, \\ London SW7 2BZ, U.K. \\ 2 Department of Physics, Chalmers University, \\ Gothenburg, Sweden \\ ${ }^{3}$ Center for Theoretical Physics, Texas A\&3M University, \\ College Station, TX 77843-4242, USA
}

\begin{abstract}
Liouville and Toda gravity theories with non-vanishing interaction potentials have spectra obtained by dividing the free-field spectra for these cases by the Weyl group of the corresponding $A_{1}$ or $A_{2}$ Lie algebra. We study the canonical transformations between interacting and free fields using the technique of intertwining operators, giving explicit constructions for the wavefunctions and showing that they are invariant under the corresponding Weyl groups. These explicit constructions also permit a detailed analysis of the operator-state maps and of the nature of the Seiberg bounds.
\end{abstract}

\footnotetext{
* Research Supported in part by the Commission of the European Communities under Contracts SC1*0394-C, SC1*-CT91-0674 and SC1*-CT92-0789.

$\dagger$ Supported in part by the U.S. Department of Energy, under grant DE-FG05-91ER40633.
} 


\section{Introduction}

Non-critical string theories achieve quantum consistency by promoting the local parameters of anomalous worldsheet symmetries to dynamical fields and then considering enlarged theories in which the anomalies are canceled via the dynamical effects of these additional fields. The treatment of the Liouville mode of ordinary noncritical string theory in such a fashion is by now familiar, dating from Polyakov's original paper on the subject [1]; for recent reviews, see Refs [2-5]. It should be kept in mind that the generic form of the trace anomaly relevant to worldsheet conformal transformations includes both a term proportional to the Euler number of the worldsheet and also a volume, or "cosmological constant" term [6]. Although the precise coefficient of the latter depends on regularisation details of the quantisation procedure, the important fact for our purposes is that this coefficient is generically non-vanishing. The specific value of this coefficient may in any case be altered by a constant shift in the Liouville field, so no specific value is physically meaningful except for its being nonvanishing. Therefore, one necessarily must deal with the full nonlinear dynamics of the Liouville mode, and not just with its contribution to the total central charge of the theory. Furthermore, owing to the inessential nature of the Liouville potential's coefficient, it is not appropriate to treat this nonlinearity in a perturbative fashion.

Extensions of bosonic string theories to models with worldsheet $W$ symmetries give rise to non-critical behaviour generalising that of the Liouville case. The possible Lagrangians for the compensating modes incorporated into the theory in order to cancel the anomalies may be determined by consistency considerations. Similarly to the Liouville case, the compensating modes taken together with the original matter fields must form a realisation of the $W$-algebra with the specific value of the central charge that is needed for overall cancellation of the anomalies. The central-charge contributions from these modes, needed also for the Virasoro subalgebra, may be obtained already at the "classical" level by including background-charge terms $\int d^{2} \sigma \sqrt{\hat{g}} R(\hat{g}) \vec{Q} \cdot \vec{\varphi}$ into the Lagrangian for the compensating modes $\vec{\varphi}$, where $\hat{g}_{i j}$ is the fiducial worldsheet metric. For gauge fields coupled to the higher-spin currents of the $W$ algebra, there are analogous background-charge terms. The $W$-algebra transformations of the $\vec{\varphi}$ may be obtained in the operator formalism by taking commutators with the $W$ generators. The interaction potential terms for the $\vec{\varphi}$ must be left invariant by these transformations. Demanding also that the classical limit as $\hbar \rightarrow 0$ be smooth, one can determine the forms of the allowed potentials.

To be specific, consider the Euclidean action for a Liouville field arising as a result of the conformal anomalies:

$$
I=\frac{1}{4 \pi} \int d^{2} \sigma \sqrt{\hat{g}}\left(\frac{1}{2}(\hat{\nabla} \varphi)^{2}+Q \varphi R(\hat{g})+\frac{4}{\gamma^{2}} e^{\gamma \phi}\right)
$$


From this, one obtains the stress tensor by varying with respect to the fiducial metric $\hat{g}_{i j}$, giving the standard result for the chiral (holomorphic) component, where $z=e^{\mathrm{i} \sigma+\tau}$,

$$
T_{z z}=T=-\frac{1}{2}(\partial \varphi)^{2}-Q \partial^{2} \varphi
$$

Using free-field commutation rules, one obtains the conformal weight of the potential $e^{\gamma \varphi}$, $\Delta=-\frac{1}{2} \gamma(\hbar \gamma+2 Q)$. Thus the condition for conformal invariance, that the potential term have weight $(1,1)$, is

$$
\gamma=\frac{1}{\hbar}\left(-Q \pm \sqrt{Q^{2}-2 \hbar}\right)
$$

where we have shown explicitly factors of $\hbar$ in order to discuss the classical limit. Demanding that $\gamma$ have a smooth limit (to the classical value $-Q^{-1}$ ) as $\hbar \rightarrow 0$, since anomaly cancellation must be carried out order by order in $\hbar$, one must select the $+\operatorname{sign}$ in (1.3) (for $Q>0$ ).

In the case of non-critical $W_{3}$ strings, two compensating-mode fields $\left(\varphi_{1}, \varphi_{2}\right)$ are needed. The chiral stress tensor in the presence of background charges $\left(Q_{1}, Q_{2}\right)$ becomes

$$
T=T_{1}+T_{2}=\left[-\frac{1}{2}\left(\partial \varphi_{1}\right)^{2}-Q_{1} \partial^{2} \varphi_{1}\right]+\left[-\frac{1}{2}\left(\partial \varphi_{2}\right)^{2}-Q_{2} \partial^{2} \varphi_{2}\right] .
$$

As in the Liouville case, potential terms $e^{\vec{\gamma} \cdot \vec{\varphi}}$ must have weight $(1,1)$ under the (left, right) Virasoro transformations. Using free-field commutation rules, the weight of an exponential $e^{\vec{\gamma} \cdot \vec{\varphi}}$ is now given by $\Delta=-\frac{1}{2} \vec{\gamma} \cdot(\hbar \vec{\gamma}+2 \vec{Q})$, so setting $\Delta=1$ yields an equation relating $\gamma_{1}$ and $\gamma_{2}$; this may be solved to give

$$
\gamma_{1}=\frac{1}{\hbar}\left(-Q_{1} \pm \sqrt{Q_{1}^{2}-2 \hbar-\hbar^{2} \gamma_{2}^{2}-2 Q_{2} \gamma_{2} \hbar}\right)
$$

Demanding that $\gamma_{1}$ have a smooth limit as $\hbar \rightarrow 0$ requires that we pick the + sign in (1.5).

In addition, one must require invariance of $\int d^{2} \sigma \sqrt{\hat{g}} e^{\vec{\gamma} \cdot \vec{\varphi}}$ under the spin-3 transformations as well. This may be achieved by requiring that the operator product of the chiral spin-3 current [13]

$$
W=\frac{1}{3}\left(\partial \varphi_{1}\right)^{3}+Q_{1} \partial \varphi_{1} \partial^{2} \varphi_{1}+\frac{1}{3} Q_{1}^{2} \partial^{3} \varphi_{1}+2 \partial \varphi_{1} T_{2}+Q_{1} \partial T_{2}
$$

with $e^{\vec{\gamma} \cdot \vec{\varphi}}$ give a total $\partial=\partial / \partial z$ derivative. Requiring this in the case of the minimal $W_{3}$ string with only the two compensating modes $\varphi_{1}$ and $\varphi_{2}$ present yields four solutions for $\gamma_{2}$,

* Strictly speaking, a satisfactory derivation of the form of the potential terms in non-critical string theories should be done on the basis of consistency requirements within the interacting theory including the potential. By requiring Virasoro covariance, this has been carried out in standard non-critical string theory [7-12], giving the same result as the free-field conformal-weight analysis summarised here. The analogous full consistency analysis of the potentials for non-critical $W$-strings remains an open problem. 
using the + solution chosen in (1.5). In order for $T$ and $W$ to form a realisation of the $W_{3}$ algebra, one must have $Q_{1}=\sqrt{3} Q_{2}$; the four solutions for $\gamma_{2}$ are then

$$
\begin{aligned}
& \gamma_{2}=\frac{1}{\hbar}\left(-Q_{2}+\sqrt{-\frac{1}{2} \hbar+\frac{5}{2} Q_{2}^{2} \mp \frac{3}{2} Q_{2} \sqrt{Q_{2}^{2}-2 \hbar}}\right) \\
& \gamma_{2}=\frac{1}{\hbar}\left(-Q_{2} \pm \sqrt{Q_{2}^{2}-2 \hbar}\right) .
\end{aligned}
$$

Requiring again a smooth classical limit as $\hbar \rightarrow 0$ eliminates two of the above four possibilities, leaving just the - solution of $(1.7 a)$ and the + solution of $(1.7 b)$. For a $W_{3}$ string with only two scalar fields $\varphi_{1}$ and $\varphi_{2}$, the conditions for anomaly cancellation require $Q_{1}=\sqrt{\frac{49}{8}}$, implying $Q_{2}=\sqrt{\frac{49}{24}}$. Consequently, the allowable potentials for the two-scalar $W_{3}$ string are [14]

$$
\begin{aligned}
V_{1} & =e^{-\frac{3}{7} Q_{1} \varphi_{1}+\frac{3}{7} Q_{2} \varphi_{2}} \\
V_{2} & =e^{-\frac{6}{7} Q_{2} \varphi_{2}} .
\end{aligned}
$$

In the context of free-field $W_{3}$ conformal field theory, the potentials $V_{1}$ and $V_{2}$ may, upon replacing the field variables $\varphi_{1}$ and $\varphi_{2}$ by field operators, be viewed as screening currents; indeed, our derivation of their structure used precisely this relation. The other two solutions of (1.7), which we have rejected as interaction potentials for the Lagrangian on the grounds of not having a smooth classical limit, also give rise to screening currents in the free-field conformal field theory. For reference, we give their structure as well:

$$
\begin{aligned}
& \tilde{V}_{1}=e^{-\frac{4}{7} Q_{1} \varphi_{1}+\frac{4}{7} Q_{2} \varphi_{2}} \\
& \tilde{V}_{2}=e^{-\frac{8}{7} Q_{2} \varphi_{2}} .
\end{aligned}
$$

In this paper, we shall principally be concerned with the two-scalar $W_{3}$ string without further matter fields. If one includes extra matter fields, one must make a choice between the multi-scalar construction of Refs $[15,16]$, which proceeds by incorporating the extra scalars into $T_{2}$ in $(1.4,1.6)$ and changing $Q_{2}$ so as to maintain the effective central charge for $T_{2}$ equal to $\frac{51}{2}$, or the construction of Ref. [17], which adds the matter fields in a separate $W_{3}$ representation in such a fashion as to make an overall nilpotent BRST operator for the whole theory. The free-field multi-scalar construction of Refs [16,14] gives rise to two screening currents only, of which one fails to have a smooth classical limit; the remaining one is $V_{2}$. In the case of the construction of Ref. [17], we find that both $V_{1}$ and $V_{2}$ in (1.8) generalise to give screening charges with smooth classical limits. In that case, one has background charges $Q_{1}$ and $Q_{2}$ for the "Toda" sector, and background charges $Q_{1}^{M}$ and $Q_{2}^{M}$ 
for the "matter" sector. The screening charges have momenta only in the Toda sector, and are given by vertex operators

$$
\tilde{V}=e^{p_{1} \varphi_{1}+p_{2} \varphi_{2}}
$$

where $p_{1} / Q_{1}=-p_{2} / Q_{2}=-\frac{1}{2}+\frac{1}{2} \sqrt{1-6 / Q_{1}^{2}}$ for the generalisation of $V_{1}$, and $p_{1}=0$, $p_{2} / Q_{2}=-1+\sqrt{1-6 / Q_{1}^{2}}$ for the generalisation of $V_{2}$. As before there are two more screening charges, corresponding to the other sign of the square root $\sqrt{1-6 / Q_{1}^{2}}$; these generalise the screening charges given by (1.9) that do not have smooth classical limits.

With the potentials (1.8) included into the Lagrangian, the two-scalar $W_{3}$ string becomes an open Toda chain theory [18]. In the rest of this paper, we shall study the spectra and dynamics of the Liouville and Toda cases, emphasising the rôle of the center-of-mass zero modes that must be treated non-perturbatively. We shall, as in many treatments of the Liouville case $[2-5]$, be concerned largely with the "minisuperspace" approximation, in which only the zero mode is kept, and no field-theory oscillator excitations are considered. A new technique that we shall bring to these much-studied questions is the approach of canonical transformations for exactly-integrable systems via intertwining operators, which has been elaborated in Refs [19-22]. The intertwining operator is a quantum-mechanical implementation of a canonical transformation, written in such a way as to give an explicitlycalculable action on wavefunctions.

In section two, we shall examine carefully the canonical transformation in the Liouville case in order to set the stage for our further discussion. For our purposes, an important fact will be that the canonical transformation between the Liouville theory and its associated free-field theory has a twofold branch structure. The branches of the transformation in the general Toda case carry a representation of the Weyl group for the underlying Lie algebra of the Toda theory, which in the Liouville case is just $A_{1}$, with Weyl group $Z_{2}$. Next, we shall introduce the quantum intertwining operator for the Liouville case, showing how the wavefunction for the Liouville zero mode can be obtained, with the Weyl-multiplet structure of the intertwining operator being reflected in the Weyl-group symmetry of the wavefunction. This makes explicit the two-to-one nature of the relation between the free theory and the Liouville theory, with two states related by the Weyl group in the free theory being mapped onto the same Liouville state. This formalism will then be compared to other quantum canonical transformations in the literature, which mainly focus on the relation between interacting-theory and free-theory field operators. We shall rederive in particular the known results for the Heisenberg representation field operator in the case of Liouville quantum mechanics $[23,24]$.

In section three, we shall compare the present intertwining-operator canonical transformation to the "operator-state map" path integral that has frequently been employed to obtain interacting-theory wavefunctions from time-asymptotic plane-wave forms of wavefunctions. We shall find, through a careful analysis of the Liouville case, that this specific 
construction introduces an asymmetry between positive and negative plane-wave momenta. This is the origin of the "Seiberg bound" for the Liouville theory, which is here seen to be a rather specific property of the operator-state map rather than an intrinsic feature of Liouville theory itself. The operator-state map for the important case of imaginary plane-wave momenta will be seen to produce in general a superposition of Liouville wavefunctions, and not just a single Liouville eigenfunction.

In section four, we shall turn our attention to the $A_{2}$ Toda theory, which as we have seen above is related to the $W_{3}$ string. Paralleling our Liouville discussion, we first shall examine the classical canonical transformations between the interacting theory and the related free theory, emphasising the Weyl-multiplet structure of the transformation. An intertwiningoperator derivation will then be given of the wavefunction for the Toda center-of-mass zero modes, giving a Weyl-symmetric result. This will establish the six-to-one nature of the map between free-theory states and Toda states.

In the conclusion, we shall discuss the implications of our results for the spectra of noncritical strings and $W$-strings. Much of the existing literature on Liouville gravity [25,7-12] has been concerned only with the relation between free and interacting-theory states in the ghost-vacuum sector. For such states, the ghosts can be completely factored out of the discussion. On the other hand, it is now well-known [26,27] that, in the free theories associated to pure Liouville gravity or Liouville gravity plus matter, there is a far richer structure of physical states involving excitations of the ghost fields. We shall see that the excited-state spectra of free Virasoro and $W_{3}$ strings have a the Weyl-multiplet structure that combines states at different ghost numbers. This suggests that the ghosts enter in an essential way into the full field-theoretic multivalued maps between the interacting and free theories in these cases.

In the appendix, we shall present variations of the intertwining-operator technique that produce forms of the Toda-theory wavefunctions that have previously appeared [28-30] in the integrable-model literature.

\section{Canonical transformations for Liouville gravity}

As discussed in the introduction, for the non-critical bosonic string the ghost-vacuum sector of the physical spectrum is described by a Liouville field theory coupled to the matter system. At the quantum level, the study of Liouville field theory is a complicated problem which has not been completely solved. However, many of the subtleties that arise are associated specifically with the zero mode of the Liouville field. This can be studied in isolation using the so-called minisuperspace approximation [2-5,31]. In this approximation, Liouville field theory reduces to Liouville quantum mechanics.

An important feature of Liouville theory is its integrability; there is a Bäcklund transformation that maps it into a completely free theory. This transformation, which can be 
implemented either at the level of the field theory or in the minisuperspace limit, renders the theory solvable, but it does not imply that it is trivial. It is not trivial because this transformation, although canonical, is not unitary. In particular it is a two-to-one map. Also, there is no vacuum state in Liouville theory since the zero-momentum limit of the physical states is not normalizable. Nevertheless, one can in principle solve the Liouville theory by mapping back from results for correlation functions in the associated free theory, using the canonical transformation. Since this transformation is not unitary, one must be careful to preserve the Hilbert-space inner product when mapping from the free to the interacting theory, by applying suitable normalization factors. Although these steps have not been completely carried out for the full field theory, we shall show that one can implement them completely at the quantum-mechanical level. ${ }^{\dagger}$

We shall principally be concerned with the zero-mode contribution to the Liouville Hamiltonian, corresponding to the minisuperspace approximation. We may split up the Liouville field and introduce a convenient rescaling by

$$
\begin{aligned}
\varphi(\tau, \sigma) & =\frac{2}{\gamma} q(\tau)+\varphi^{\mathrm{osc}}(\tau, \sigma), \\
\oint d \sigma \varphi^{\mathrm{osc}} & =0 .
\end{aligned}
$$

Then, ignoring for the moment the background-charge term, the action for the minisuperspace mode is that for Liouville quantum mechanics,

$$
\pi \gamma I \underset{\mathrm{mss}}{\longrightarrow} I_{\mathrm{L}}=\frac{1}{2} \int d \tau\left(\dot{q}^{2}+e^{2 q}\right)
$$

The background-charge term affects the relation between the momentum of this Liouville system and the momentum customarily used to classify states in conformal field theory, which is the parameter $\alpha$ in a vertex operator $e^{\alpha \varphi(z, \bar{z})}$, where $z=e^{\mathrm{i} \sigma+\tau}$. Owing to the presence of the background charge in (1.2), the conformal transformation of $\varphi$ from the $(z, \bar{z})$ variables to the $(\tau, \sigma)$ variables induces an extra term in the transformation of $\partial_{z} \varphi$, i.e. $\partial_{z} \varphi \rightarrow\left(\partial_{w} \varphi-Q\right) / z$, where $w=\mathrm{i} \sigma+\tau$. As a consequence, the total momentum inserted by a vertex operator $e^{\alpha \varphi(z, \bar{z})}$ is shifted with respect to the Liouville momentum [2-5]:

$$
\mathrm{i} p_{\varphi}=\alpha+Q
$$

Accordingly, we shall have to take this shift into account in comparing Liouville quantum mechanics to non-critical string theory.

$\dagger$ There has been a considerable body of work on quantum Bäcklund transformations for Liouville theory (e.g., $[11,32,33])$. Much of this has been concerned with the relations between free-theory and interactingtheory field operators. Here, we are mostly concerned with the mapping of wavefunctions between the free and interacting theories. We shall, however, discuss the transformation of field operators from our point of view later in this section. 


\subsection{Classical Liouville Mechanics}

We begin our discussion of the canonical transformations to a free theory at the classical level, letting the time parameter be denoted now by $t$. The Hamiltonian for the minisuperspace mode following from $(2.2)$ is

$$
H_{\mathrm{L}}=\frac{1}{2}\left(p^{2}+e^{2 q}\right)
$$

where $p(t)$ is the momentum canonically conjugate to $q(t)$. Hamilton's equations give the equations of motion $\dot{q}=p$ and $\dot{p}=-e^{2 q}$, so we have $\ddot{q}+e^{2 q}=0$. Thus, from (2.1.1) we have a first integral $\dot{q}^{2}+e^{2 q}=2 E$, where the energy $E$ is a constant. Performing the remaining integral, we obtain

$$
q=-\ln \left(\frac{1}{\tilde{p}} \cosh (\tilde{p} t)\right) .
$$

Here $\tilde{p}$ is an arbitrary constant, related to $E$ by $E=\frac{1}{2} \tilde{p}^{2}$; the second constant of integration has been absorbed into the choice of origin for $t$. The solution may be written in the form

$$
\begin{aligned}
e^{-q} & =\frac{1}{\tilde{p}} \cosh \tilde{q} \\
p & =-\tilde{p} \tanh \tilde{q},
\end{aligned}
$$

where $\tilde{q}=\tilde{p} t$. In fact, these equations can be viewed as a canonical transformation from the interacting Liouville system, with phase-space coordinates $(q, p)$, to a free system with phase-space coordinates $(\tilde{q}, \tilde{p})$ and Hamiltonian

$$
\tilde{H}_{\mathrm{L}}=\frac{1}{2} \tilde{p}^{2}
$$

Under this transformation, the solution $\tilde{p}=$ constant, $\tilde{q}=\tilde{p} t$ for the free system is mapped to the solution (2.1.2) for the Liouville system.

The free Hamiltonian (2.1.4) has a $Z_{2}$ symmetry under the discrete canonical transformation

$$
(\tilde{q}, \tilde{p}) \longrightarrow(-\tilde{q},-\tilde{p}) .
$$

This $Z_{2}$ can be understood as the Weyl group of the $A_{1}=S L(2, \mathbb{R})$ algebra underlying the Liouville theory $\ddagger$ Combined with the canonical transformation (2.1.3), this symmetry gives another form for the canonical transformation from the free to the interacting theory, namely

$$
\begin{aligned}
e^{-q} & =-\frac{1}{\tilde{p}} \cosh \tilde{q} \\
p & =-\tilde{p} \tanh \tilde{q} .
\end{aligned}
$$

$\ddagger$ This Weyl-group symmetry is also the discrete residuum of a full $S L(2, \mathbb{R})$ invariance of the Bäcklund transformation for Liouville field theory [7]. 
Note that (2.1.3) and (2.1.6) have different classical domains of applicability. Eq. (2.1.3) only makes sense classically for $\tilde{p}>0$, and (2.1.6) only makes sense for $\tilde{p}<0$. The transformation (2.1.3) maps a right-moving solution of the free theory, having positive momentum $\tilde{p}$ and moving from $\tilde{q}=-\infty$ at $t=-\infty$ to $\tilde{q}=+\infty$ at $t=+\infty$, into a solution of the Liouville theory that starts from $q=-\infty$ at $t=-\infty$, bounces off the potential and goes to $q=-\infty$ again at $t=+\infty$. For a left-moving solution of the free theory, we must use the second canonical transformation (2.1.6). This then maps into the identical solution of the Liouville theory. Combining the domains of (2.1.3) and (2.1.6), we have essentially only one canonical transformation from the free to the interacting theory. One may summarise this by writing (2.1.3) and (2.1.6) together as

$$
\begin{aligned}
e^{-q} & =\frac{1}{|\tilde{p}|} \cosh \tilde{q} \\
p & =-\tilde{p} \tanh \tilde{q} .
\end{aligned}
$$

Upon performing an inverse canonical transformation from the interacting Liouville theory to the free theory, one encounters a branch structure: there is a choice, for given $(q, p)$, of obtaining either $(\tilde{q}, \tilde{p})$ or $(-\tilde{q},-\tilde{p})$, corresponding to the choice of inverting (2.1.3) or (2.1.6) above. This branch structure is the basic expression of the two-to-one nature of the map from the free to the interacting Liouville theory.

A further reflection of the two-to-one nature of the Liouville free-field map is that, if we consider the asymptotic limit of the transformation (2.1.7), we see that when $q$ is large and negative we have $q \rightarrow \pm \tilde{q}+\ln (2|\tilde{p}|)$, so both asymptotic regions of the free theory map into the same asymptotic region of the Liouville theory.

This asymptotic limit in $q$ corresponds to the behaviour of the classical solutions as $t \rightarrow \pm \infty$. Specifically, for $t \rightarrow-\infty$, and $\tilde{p}>0$, we find that $e^{-\tilde{q}}=e^{-\tilde{p} t}$ dominates $e^{\tilde{q}}=e^{\tilde{p} t}$, so from (2.1.3) we find $p \rightarrow \tilde{p}$ and $e^{-q} \rightarrow(2 \tilde{p})^{-1} e^{-\tilde{q}}$. Thus, the time-asymptotic momentum of the interacting theory gives the value of the free momentum $\tilde{p}$. For $t \rightarrow-\infty$ with $\tilde{p}<0$, $e^{\tilde{q}}$ dominates $e^{-\tilde{q}}$ and (2.1.6) implies $p \rightarrow-\tilde{p}, e^{-q} \rightarrow-(2 \tilde{p})^{-1} e^{\tilde{q}}$. It is thus clear that the cases $\tilde{p}>0$ and $\tilde{p}<0$ give rise to the same Liouville motion $q(t)$, but with an opposite identification of the free momentum $\tilde{p}$ in terms of the asymptotic $p(t)$ as $t \rightarrow-\infty$.

The limit as $t \rightarrow+\infty$ may be handled similarly, giving $e^{-q} \rightarrow \pm(2 \tilde{p})^{-1} e^{ \pm \tilde{q}} ; p \rightarrow \mp \tilde{p}$ for $\tilde{p} \gtrless 0$. Note that the $t \rightarrow \pm \infty$ limits of the Liouville momentum $p(t)$ differ precisely by a Weyl-group reflection, so the conservation of $\tilde{p}$ in the free theory implies the conservation of asymptotic values of $p(t)$ modulo the Weyl group.

\subsection{Quantum Liouville Mechanics}

The canonical transformation between the classical Liouville and free theories discussed in the previous subsection can be generalised to the quantum level. We shall use the technique of intertwining operators [19-22], which implements the canonical transformation on 
quantum operators and also on wavefunctions. The canonical transformation will be generated by an intertwining operator $C$, which maps any quantum operator $A$ in the interacting theory into the corresponding operator $\tilde{A}$ in the free theory according to the rule:

$$
C A C^{-1}=\tilde{A}
$$

In particular, we have the transformation $C H_{\mathrm{L}} C^{-1}=\tilde{H}_{\mathrm{L}}$ from the interacting to the free Hamiltonian. As a consequence, wave functions $\psi$ and $\tilde{\psi}$ of the interacting and free theories are related by

$$
\psi=C^{-1} \tilde{\psi}
$$

In order to interpret the latter, it is necessary to break up the canonical transformation into a sequence of elementary canonical transformations, each of which has a well-defined action on wavefunctions.

Starting from the Liouville Hamiltonian (2.1.1), we perform the following sequence of elementary canonical transformations:

$\begin{array}{llll}{[\mathrm{L} 1]} & \mathcal{P}_{\ln q}: & q \mapsto \ln q, & p \mapsto q p \\ {[\mathrm{~L} 2]} & \mathcal{I}: & q \mapsto p, & p \mapsto-q \\ {[\mathrm{~L} 3]} & p^{-1}: & q \mapsto p^{-1} q p=q+\mathrm{i} p^{-1}, & p \mapsto p \\ {[\mathrm{~L} 4]} & \mathcal{P}_{\sinh q}: & q \mapsto \sinh q, & p \mapsto \frac{1}{\cosh q} p\end{array}$

Acting on a wavefunction $\psi(q)$, the operator $\mathcal{P}_{f(q)}[\mathrm{L} 1, \mathrm{~L} 4]$ associated with the point transformation $q \mapsto f(q)$ makes the replacement $\mathcal{P}_{f(q)} \psi(q)=\psi(f(q))$. The operator $\mathcal{I}$ [L2], associated with the discrete canonical transformation $(q, p) \mapsto(p,-q)$, is realised on functions of $q$ as a Fourier transform ${ }^{\S}, \mathcal{I} \psi(q)=\frac{1}{\sqrt{2 \pi}} \int d q e^{\mathrm{i} k q} \psi(q)$. Its inverse $\mathcal{I}^{-1}$ is realised as an inverse Fourier transform. Finally, when acting on a function of $q, p$ is realised by differentiation, $p \psi(q)=-\mathrm{i} \frac{\partial}{\partial q} \psi(q)$, and its inverse $p^{-1}$ [L3] is realised by integration and multiplication by i.

Wrapping the stages [L1-L4] together, the full relation between the interacting and the free variables may be written

$$
\begin{aligned}
e^{-q} & =\frac{1}{\tilde{p}} \cosh \tilde{q} \\
p & =-\tanh (\tilde{q}) \tilde{p} .
\end{aligned}
$$

Note that, although these take a form similar to the classical canonical transformation (2.1.3), Eq. (2.2.3) is a full quantum-mechanical relation, and the indicated ordering of the operators $(\tilde{q}, \tilde{p})$ in it is essential.

\footnotetext{
$\S$ The contours of integration in the integral transforms $\mathcal{I}$ and $\mathcal{I}^{-1}$ must be chosen so that boundary terms vanish when integrating by parts in verifying the transformations of $q$ and $p$. In particular, this can allow limits of integration other than $\pm \infty$.
} 
Taking into account the commutation relation $[p, q]=-\mathrm{i}$, one can easily perform the sequence of canonical transformations [L1-L4] on the Liouville Hamiltonian:

$$
\begin{aligned}
2 H_{\mathrm{L}} & =p^{2}+e^{2 q} \\
& \mapsto(q p)^{2}+q^{2}=q^{2} p^{2}-\mathrm{i} q p+q^{2} \\
& \mapsto p^{2} q^{2}+\mathrm{i} p q+p^{2} \\
& \mapsto p q^{2} p+\mathrm{i} q p+p^{2}=\left(1+q^{2}\right) p^{2}-\mathrm{i} q p=\left[\left(1+q^{2}\right)^{\frac{1}{2}} p\right]^{2} \\
& =\tilde{p}^{2}=2 \tilde{H}_{\mathrm{L}} .
\end{aligned}
$$

The inverse intertwining operator $C^{-1}$ is given by

$$
C^{-1}=\mathcal{P}_{e^{q}} \mathcal{I}^{-1} p \mathcal{P}_{\operatorname{arcsinh} q}
$$

Using it, we obtain the Liouville wavefunction starting from an eigenfunction $e^{\mathrm{i} k \tilde{q}}$ of the free Hamiltonian. Evaluating the sequence of operations in $C^{-1} e^{\mathrm{i} k \tilde{q}}$, we have, leaving a $k$-dependent normalization factor $N_{k}$ undetermined for the moment,

$$
\psi_{k}(q)=N_{k} \mathcal{P}_{e^{q}} \mathcal{I}^{-1} p \mathcal{P}_{\frac{1}{2}\left(q-q^{-1}\right)}^{-1} q^{\mathrm{i} k}
$$

since $\mathcal{P}_{\operatorname{arcsinh} q}=\mathcal{P}_{\frac{1}{2}\left(q-q^{-1}\right)}^{-1} \mathcal{P}_{e^{q}}^{-1}$. Next, we let $u=\frac{1}{2}\left(y-y^{-1}\right)$ define $y(u)$ implicitly. In order to reproduce the transformation $\mathcal{P}_{\operatorname{arcsinh}}$, it is necessary to choose as solution the branch $y=u+\sqrt{u^{2}+1}$. Then we have

$$
\begin{aligned}
\psi_{k}(q) & =\frac{-\mathrm{i} N_{k}}{\sqrt{2 \pi}} \mathcal{P}_{e^{q}} \int_{-\infty}^{\infty} d u e^{-\mathrm{i} q u} \frac{\partial}{\partial u}(y(u))^{\mathrm{i} k} \\
& =\frac{k N_{k}}{\sqrt{2 \pi}} \int_{0}^{\infty} d y e^{-\frac{\mathrm{i}}{2} e^{q}\left(y-y^{-1}\right)} y^{\mathrm{i} k-1} .
\end{aligned}
$$

Strictly speaking, this integral needs to be regularized, e.g. by rotating its integration contour by an angle $\epsilon$ into the lower half plane and by taking $y-y^{-1}$ to mean $y+e^{-i \pi} y^{-1}$. Evaluating the integral, we obtain [34]

$$
\psi_{k}(q)=\frac{2 k N_{k}}{\sqrt{2 \pi}} e^{\frac{\pi k}{2}} K_{\mathrm{i} k}\left(e^{q}\right)
$$

where $K_{\mathrm{i} k}$ is a modified Bessel function.

Note that the wavefunction (2.2.8) is not yet correctly normalized. This is more than just an issue of a missing factor of $\frac{1}{\sqrt{2 \pi}}$ for the initial free-field plane wave. The intertwining operator $C$ correctly generates a canonical transformation at the quantum level, but this

ฯ A related derivation may be found in the appendix of Ref. [20]. 
transformation was never required to be unitary. Instead, we are making a more general similarity transformation of the form (2.2.1) on operators in the theory. One may calculate the effect of the transformation $C$ on the Hilbert-space inner product, which is not invariant under $C$ because $C$ is not unitary. In this way, we may learn the ( $k$-dependent) factor needed to convert (2.2.8) into a fully-normalized wavefunction. The final result after normalization is:

$$
\psi_{k}(q)=\frac{1}{\pi} \sqrt{2 k \sinh (\pi k)} K_{\mathrm{i} k}\left(e^{q}\right) .
$$

This wavefunction satisfies, for all complex values* of $k$, the physically-required boundary condition of falling away to zero as $q \rightarrow \infty$, i.e. under the Liouville potential. Of course, the time-independent Schrödinger equation is a second-order differential equation and should have a second solution which may, from general experience with quantum mechanics, be expected not to satisfy the boundary conditions as $q \rightarrow \infty$. This second, physically-rejected, solution to Schrödinger's equation may also be obtained via an alternative intertwiningoperator transformation starting from the plane-wave eigenfunctions of the free Hamiltonian $\tilde{H}_{\mathrm{L}}$. The existence of another transformation that maps $H_{\mathrm{L}}$ into $\tilde{H}_{\mathrm{L}}$ can easily be seen in the sequence of transformations (2.2.4), where the $\mathcal{I}$ transformation [L2] acts upon an expression $q^{2} p^{2}-\mathrm{i} q p+q^{2}$ that is already a polynomial of even order and hence is invariant under an extra sign-flip transformation $(q, p) \mapsto(-q,-p)$, immediately preceding [L2]. Consequently, the transformation to the free Hamiltonian $\tilde{H}_{\mathrm{L}}$ may equally well be done by interchanging $\mathcal{I}$ with $\mathcal{I}^{-1}$. Although this has no effect on the sequence of stages (2.2.4) to the free Hamiltonian, the replacement of $\mathcal{I}^{-1}$ by $\mathcal{I}$ in the inverse transformation

$$
C^{\prime-1} \equiv \mathcal{P}_{e^{q}} \mathcal{I} p \mathcal{P}_{\operatorname{arcsinh} q}
$$

leads ${ }^{\dagger}$ to an un-normalizable wavefunction

$$
\psi_{k}^{\prime}(q) \propto K_{\mathrm{i} k}\left(e^{\mathrm{i} \pi} e^{q}\right) .
$$

This may be expressed in terms of the independent modified Bessel function $I$ by use of the relation

$$
K_{\mathrm{i} k}\left(e^{\mathrm{i} \pi} e^{q}\right)=e^{\pi k} K_{\mathrm{i} k}\left(e^{q}\right)-\mathrm{i} \pi I^{\mathrm{i} k}\left(e^{q}\right) .
$$

Note that this solution of Schrödinger's equation fails rather spectacularly to obey the boundary condition of dying away under the potential, because $I_{\mathrm{i} k}\left(e^{q}\right)$ diverges asymptotically in

* The modified Bessel function $K_{\mathrm{i} k}(z)$ is, for fixed argument $z \neq 0$, an entire function of $k$; the same is true for the normalized wavefunction (2.2.9). Thus, although integral representations of Bessel functions such as (2.2.7) may have limited domains of convergence, the result (2.2.8) can be analytically continued throughout the complex $k$ plane.

$\dagger$ In order to obtain a solution independent from (2.2.7), the integrand in (2.2.10) should be taken to be $\exp \left(\frac{1}{2} \mathrm{i} e^{q}\left(y+e^{-\mathrm{i} \pi} y^{-1}\right)\right) y^{\mathrm{i} k-1}$. The choice of contour needed for convergence involves subtleties that we will not address in this paper; but $c f$. Ref. [34]. 
the limit $q \rightarrow \infty$ like $\exp \left(e^{q}-q / 2\right)$. In the sequel, we shall confine our attention to the canonical transformation effected by (2.2.4) and the corresponding wavefunction $(2.2 .7,2.2 .9)$.

The quantum Liouville wavefunction is invariant under the $Z_{2}$ Weyl group of $S U(2)$, as is manifest in the fully-normalized form (2.2.9), since the modified Bessel function $K_{\mathrm{i} k}$ is symmetric under $k \rightarrow-k$. Thus, the two-to-one character of the free-field to Liouville map that we encountered at the classical level is equally present at the quantum level: both free-field wavefunctions $e^{\mathrm{i} k \tilde{q}}$ and $e^{-\mathrm{i} k \tilde{q}}$ map to the same Liouville wavefunction. One can equivalently express this in terms of a $Z_{2}$ branch structure for the inverse map from the interacting wavefunction to the free wavefunctions: one is then faced with a choice of mapping $\psi_{k}\left(e^{q}\right)$ either back to $e^{\mathrm{i} k \tilde{q}}$ or to $e^{-\mathrm{i} k \tilde{q}}$.

In order to set the stage for our later Toda discussion in section 4, we note also that the Weyl-group symmetry can already be seen in the un-normalized form (2.2.7) of the wavefunction. Let us first rewrite the integral in a manifestly convergent form by rotating the contour of integration so as to run along the negative imaginary axis, making at the same time the change of variables $y=e^{-\mathrm{i} \pi / 2} w$. We thus obtain

$$
\psi_{k}(q)=\frac{N_{k} k e^{\frac{\pi k}{2}}}{\sqrt{2 \pi}} \int_{0}^{\infty} d w e^{-\frac{1}{2} e^{q}\left(w+w^{-1}\right)} w^{\mathrm{i} k-1}
$$

At this stage, we may make a change in the variable of integration $w \rightarrow w^{-1}$, yielding

$$
\psi_{k}(q)=\frac{N_{k} k e^{\frac{\pi k}{2}}}{\sqrt{2 \pi}} \int_{0}^{\infty} d w e^{-\frac{1}{2} e^{q}\left(w^{-1}+w\right)} w^{-\mathrm{i} k-1} .
$$

This change of integration variable thus has the same effect on the integral as a Weyl-group transformation $k \rightarrow-k$. Consequently, application of a Weyl-group transformation to the un-normalised wavefunction affects only the prefactor $k e^{\frac{\pi k}{2}}$ of the integral in $(2.2 .14)$; upon normalization, this is exchanged for the Weyl-symmetric factor $(2 \pi)^{-1}(2 k \sinh (\pi k))^{1 / 2}$.

Note that the form (2.2.13) of the integral representation for $\psi_{k}(q)$ makes it manifest that the wavefunction falls off rapidly in the classically-forbidden region $q>>0$ under the Liouville-potential "mountain." In fact, from the asymptotic behaviour of the $K$ Bessel function, one sees that it falls off as $\exp \left(-e^{q}-\frac{1}{2} q\right)$ at large positive $q$.

It is also worth noting that the manifestly-convergent integral (2.2.13) for the wavefunction may itself be obtained directly from an intertwining-operator realisation of a canonical transformation. This transformation differs from the classically-motivated one [L1-L4] by the replacement of the final [L4] point transformation by $\left[\mathrm{L} 4^{\prime}\right]: \mathcal{P}_{-i \cosh q}$. This may also be viewed as an additional shift of the free-field variable, $\tilde{q} \mapsto \tilde{q}-\frac{1}{2} \mathrm{i} \pi$. This additional transformation is not appropriate for the variables of the classical theory, which must be 
real, but there is no reason to exclude it at the quantum level. Combining the elementary transformations into an overall canonical transformation, one has

$$
\begin{aligned}
e^{-q} & =\frac{-\mathrm{i}}{\tilde{p}} \sinh \tilde{q} \\
p & =-\operatorname{coth}(\tilde{q}) \tilde{p},
\end{aligned}
$$

which should be compared with the classically-implementable transformation (2.2.3).

Unlike (2.2.3), the form (2.2.15) for the transformation of the quantum operators $(\tilde{p}, \tilde{q})$ is manifestly Weyl-group symmetric. This does not necessarily mean that the intertwiningoperator transformation of the wavefunction is Weyl-group symmetric. It does imply, however, that a Weyl-group transformation of the wavefunction (2.2.13) produces a variation that is at worst an overall rescaling. For intertwining operators $C$ and $C^{\prime}$ differing by the application of a Weyl-group transformation, the invariance of (2.2.15) implies that the operator $C^{-1} C^{\prime}$ must commute with $\tilde{p}$ and $\tilde{q}$. Thus it follows that [21]

$$
C^{-1}=\mu\left(C^{\prime}\right)^{-1}, \quad \mu \in \mathbb{C}
$$

so the wavefunctions can differ at most by a scale factor $\mu$.

In non-critical string theory, the Liouville momentum of physical operators and states is given complex values. In the limit as $q \rightarrow-\infty$, where the Liouville potential dies away, the wavefunction (2.2.9) becomes asymptotically

$$
\psi_{k}(q) \underset{q \rightarrow-\infty}{\longrightarrow}-\sqrt{\frac{2 k}{\sinh (\pi k)}} \sin [k(q-\ln 2)] .
$$

Thus, if we set $k=k_{\mathrm{R}}+\mathrm{i} k_{\mathrm{I}}$, where $k_{\mathrm{R}}$ and $k_{\mathrm{I}}$ are the real and imaginary parts of $k$, this means that the asymptotic behaviour of the wavefunctions as $q \rightarrow-\infty$ is of the form

$$
\psi_{k}(q) \sim e^{-\left|k_{\mathrm{I}}\right|(q-\ln 2)} e^{\mathrm{i} s\left(k_{\mathrm{I}}\right) k_{\mathrm{R}}(q-\ln 2)},
$$

where $s\left(k_{\mathrm{I}}\right)=k_{\mathrm{I}} /\left|k_{\mathrm{I}}\right|$. In particular, we see that the wavefunctions diverge exponentially at large negative $q$, regardless of the sign of the imaginary part of $k$. This means that when one maps from the free theory, the Liouville wavefunction diverges at large negative $q$ regardless of whether the free wavefunction diverges exponentially at large negative or at large positive $\tilde{q}$.

In order to show the relation between the intertwining-operator approach to canonical transformations as used in this paper and the more familiar transformations between the operators of the free and interacting theories, we may use our results to rederive the Heisenberg-representation time evolution of the quantum-mechanical Liouville operator $e^{-q}$ $[23,24]$. In the full Liouville field theory, similar relations between interacting-theory and 
free-theory quantum operators assume a considerable importance because there is no a priori well-defined mode expansion of the interacting Liouville field to use when one wishes to include the oscillator modes in going beyond the minisuperspace approximation. Consequently, one needs to map the mode expansion of the free field into one for the interacting field via a canonical transformation, demanding in the process covariance under the Virasoro algebra and also locality [7-12]. It remains an important task for the future to develop the present quantum-mechanical intertwining-operator techniques so as to give a treatment of these classic problems in the full Liouville quantum field theory.

In the Heisenberg representation, the operator $e^{-q(t)}$ evolves according to

$$
e^{-q(t)}=e^{\mathrm{i} H_{\mathrm{L}}\left(t-t_{0}\right)} e^{-q\left(t_{0}\right)} e^{-\mathrm{i} H_{\mathrm{L}}\left(t-t_{0}\right)},
$$

i.e. it is propagated by the interacting-theory Hamiltonian. We may use the intertwining operator $C=\mathcal{P}_{\sinh q} p^{-1} \mathcal{I} \mathcal{P}_{\ln q}$ and its inverse $C^{-1}=\mathcal{P}_{e^{q}} \mathcal{I}^{-1} p \mathcal{P}_{\operatorname{arcsinh} q}$ to write (2.2.19) as

$$
e^{-q(t)}=C^{-1} e^{\frac{\mathrm{i}}{2} \tilde{p}^{2}\left(t-t_{0}\right)} C e^{-q\left(t_{0}\right)} C^{-1} e^{-\frac{\mathrm{i}}{2} \tilde{p}^{2}\left(t-t_{0}\right)} C .
$$

In order to evaluate (2.2.20), we begin in the center of this nested expression and first evaluate $C e^{-q\left(t_{0}\right)} C^{-1}$. In evaluating the free-field map for operators, the normalization issue that one must face in evaluating the map for wavefunctions is not relevant, since normalization factors cancel out in calculating $C A C^{-1}$. Performing the nested components of the transformation in the sequence [L1-L4], we obtain the result (2.2.3) for the canonical pair of operators at time $t_{0}$ :

$$
\begin{aligned}
e^{-q\left(t_{0}\right)} & =\frac{1}{\tilde{p}\left(t_{0}\right)} \cosh \tilde{q}\left(t_{0}\right) . \\
p\left(t_{0}\right) & =-\left(\tanh \tilde{q}\left(t_{0}\right)\right) \tilde{p}\left(t_{0}\right) .
\end{aligned}
$$

Evolving (2.2.21) forward in time using the free-variable evolution operator, we obtain

$$
\begin{aligned}
& e^{\frac{\mathrm{i}}{2} \tilde{p}^{2}\left(t-t_{0}\right)} \frac{1}{\tilde{p}\left(t_{0}\right)} \cosh \tilde{q}\left(t_{0}\right) e^{-\frac{\mathrm{i}}{2} \tilde{p}^{2}\left(t-t_{0}\right)}=\frac{1}{\tilde{p}\left(t_{0}\right)} \cosh \left(\tilde{q}\left(t_{0}\right)+\tilde{p}\left(t_{0}\right)\left(t-t_{0}\right)\right) \\
& =e^{-\frac{\mathrm{i}}{2}\left(t-t_{0}\right)} \frac{1}{\tilde{p}\left(t_{0}\right)}\left[\cosh \tilde{q}\left(t_{0}\right) \cosh \left(\tilde{p}\left(t_{0}\right)\left(t-t_{0}\right)\right)+\sinh \tilde{q}\left(t_{0}\right) \sinh \left(\tilde{p}\left(t_{0}\right)\left(t-t_{0}\right)\right)\right]
\end{aligned}
$$

where we have used the Baker-Campbell-Hausdorff formula $e^{A+B}=e^{A} e^{-\frac{1}{2}[A, B]} e^{B}$, which holds when $[A,[A, B]]=0=[B,[A, B]]$. Completing the nested sequence of transformations of Eq. (2.2.20) by applying $C^{-1}$ on the left and $C$ on the right, we end up with the Heisenberg representation result of Refs [23,24].

$$
e^{-q(t)}=e^{-q\left(t_{0}\right)-\frac{\mathrm{i}}{2}\left(t-t_{0}\right)}\left(\cosh \left(\sqrt{2 H_{\mathrm{L}}}\left(t-t_{0}\right)\right)-p\left(t_{0}\right) \frac{1}{\sqrt{2 H_{\mathrm{L}}}} \sinh \left(\sqrt{2 H_{\mathrm{L}}}\left(t-t_{0}\right)\right)\right),
$$


where we have used the quantum relation $\sqrt{2 H_{\mathrm{L}}}=e^{q}\left(1+\left(e^{-q} p\right)^{2}\right)^{1 / 2}$, which may be checked by verifying that its square correctly yields $2 H_{\mathrm{L}}=p^{2}+e^{2 q}$.

To conclude this section, we compare the present intertwining-operator approach to that of a related implementation of canonical transformations [33], which emphasises the rôle of the classical generating function $F(q, \tilde{q})$. At the classical level, the momenta can be derived from the transformation formulas

$$
p=\frac{\partial F}{\partial q}, \quad \tilde{p}=-\frac{\partial F}{\partial \tilde{q}}
$$

these equations implicitly determine $(\tilde{q}, \tilde{p})$ in terms of $(q, p)$. For the full Liouville field theory, the generating function is

$$
F(\phi, \tilde{\phi})=\int d \sigma\left(\phi \partial_{\sigma} \tilde{\phi}-e^{\phi} \sinh \tilde{\phi}\right)
$$

which in the minisuperspace limit $\phi(\tau, \sigma) \rightarrow q(t=\tau)$ becomes $F(q, \tilde{q})=-e^{q} \sinh \tilde{q}$ and consequently (2.2.24) reproduces our classical canonical transformation (2.1.3).

As we have noted earlier, the full quantum canonical transformation (2.2.3) takes a form similar to the classical transformation (2.1.3), but it is essential to keep track of operator ordering at the quantum level. Starting from (2.2.24), it is not always clear how one should generalise the transformation generated by $F(q, \tilde{q})$ to the quantum case. One way to do this [33] is to require that both the interacting-theory and free-theory Hamiltonians, considered as differential operators in $q$ and $\tilde{q}$, have the same action on $e^{\mathrm{i} F(q, \tilde{q})}$ :

$$
H_{\mathrm{L}}(q, p) e^{\mathrm{i} F(q, \tilde{q})}=\tilde{H}_{\mathrm{L}}(\tilde{q}, \tilde{p}) e^{\mathrm{i} F(q, \tilde{q})} .
$$

That this holds for the Liouville generator (2.2.25) is something of an accident; in general, one might expect $F(q, \tilde{q})$ to acquire quantum corrections, and to reduce to the classical expression only in the limit $\hbar \rightarrow 0$.

Once one has found a functional $F(q, \tilde{q})$ with the property $(2.2 .26)$, one can relate the interacting-theory and the free-theory wavefunctions. If one starts from an eigenstate $\tilde{\psi}(\tilde{q})$ of the free Hamiltonian $\tilde{H}_{\mathrm{L}}$ with eigenvalue $E$ and constructs the wavefunction

$$
\psi_{\mathrm{L}}(q)=\int d \tilde{q} e^{i F(q, \tilde{q})} \tilde{\psi}(\tilde{q})
$$

a consequence of (2.2.26) is that (2.2.27) is an eigenfunction of $H_{\mathrm{L}}$ with the same eigenvalue $E$. At this point, the correspondence to our intertwining-operator formulation becomes clear. The transformation (2.2.27) should be compared to the application of $C^{-1}(2.2 .5)$ to a free wavefunction, yielding the relation

$$
C^{-1}=\int d \tilde{q} e^{i F(q, \tilde{q})}
$$


This relation may be confirmed explicitly by a change of variables in (2.2.7). Thus the procedure of Ref. [33] is an alternative way of looking at the map from the free-theory wavefunction to the interacting-theory wavefunction. The disadvantage of this procedure as compared to the intertwining-operator approach adopted in this paper is that, by concentrating on the accumulated form of the canonical transformation on wavefunctions, one loses sight of the delicate operator orderings in the quantum transformation of the canonical operator pair $(q, p)$, which we obtained in (2.2.3). Also, if it had turned out that the classical generator (2.2.25) had not itself satisfied (2.2.26), it would not have been clear how to add $\hbar$-dependent corrections in order to make it do so.

\section{The operator-state map and the Seiberg bound}

In the previous section, we explored the quantum implementation of the canonical transformation between interacting and free-field Liouville wavefunctions. At this point, we wish to explain how this procedure differs from a construction frequently given for interactingtheory wavefunctions in the string-theory literature [2-5]. This latter construction is motivated by the relation between operators and states in conformal field theory. For a holomorphic conformal-field operator $\mathcal{O}(\varphi(z))$ in a free-field theory with a state-space vacuum $|0\rangle$, a physical state associated to $\mathcal{O}(\varphi(z))$ is obtained by taking the $\operatorname{limit} \lim _{z \rightarrow 0} \mathcal{O}(\varphi(z))|0\rangle$. In interacting field theories such as Liouville theory or Toda theory which do not have normalizable vacuum states in their spectra, such a construction is not appropriate. Instead, many authors use a path-integral construction of physical states "associated" to the operator $\mathcal{O}$. Without a vacuum, there is not really a proper operator formalism for creating physical states. In the path-integral formulation, $\mathcal{O}$ is not actually an operator, but becomes instead a functional of the integration variables in the path integral. Using now and for the rest of this section a Minkowskian world-sheet signature, the interacting-theory wavefunction related to $\mathcal{O}$ is given by the construction

$$
\psi_{\mathcal{O}}(\varphi(\sigma))=\int_{\mathcal{D} ;\left.\xi\right|_{\partial \mathcal{D}}=\varphi(\sigma)}\left[d \xi\left(\sigma_{i}\right)\right] e^{-\mathrm{i} I_{\mathrm{L}}} \mathcal{O}(\xi)
$$

The domain of integration $\mathcal{D}$ for $[d \xi]$ in (3.1) is over a surface extending from negative temporal infinity, bounded by an end loop $\partial \mathcal{D}$ on which Dirichlet boundary conditions $\left.\xi\right|_{\partial \mathcal{D}}=$ $\varphi(\sigma)$ are imposed. The factor of $\mathcal{O}$ in $(3.1)$ is a local insertion made at negative temporal infinity, and the action $I_{\mathrm{L}}$ in the exponential weighting the path integral is taken to be the action for the fully-interacting theory.

In analogy with the vertex operators of free-field theory, the local functional $\mathcal{O}$ in $(3.1)$ is generally taken to have the form of a free-field wave function. Specifically, for the Liouville theory, one takes $e^{\mathrm{i} p \xi\left(\sigma_{i}^{*}\right)}$, where $\sigma_{i}^{*}$ is at negative temporal infinity. In the implementation of 
the operator-state map for a noncritical string theory, the action $I$ contains a backgroundcharge term as in (1.1). The integration over the constant mode of $\xi$ then produces a shift in the momentum $p$ by a factor of $\mathrm{i} Q$, as we have already discussed in (2.3). Aside from this shift and the related choice of a relevant domain for the momentum $p$, the discussion of the operator-state map in non-critical string theory may be reduced to a discussion in Liouville field theory, and then in the minisuperspace approximation to one in Liouville quantum mechanics.

In the minisuperspace approximation, the field integration variable $\xi\left(\sigma_{i}\right)$ in the path integral (3.1) reduces to a quantum-mechanical variable $\xi(t)$, replacing as before $\tau$ by $t$ in the quantum-mechanical case. In this limit, dropping the oscillator modes in the decomposition (2.1), the path integral (3.1) becomes

$$
\psi_{\mathcal{O}}(q(t))=\lim _{t_{0} \rightarrow-\infty} \int_{t_{0}<t^{\prime}<t ;\left.\xi\right|_{t}=q(t)}\left[d \xi\left(t^{\prime}\right)\right] \quad e^{-\mathrm{i} I_{\mathrm{L}}} \mathcal{O}\left(\xi\left(t_{0}\right)\right),
$$

where the functional integration is over variables $\xi\left(t^{\prime}\right), t_{0}<t^{\prime}<t$, with each $\xi\left(t^{\prime}\right)$ running from $-\infty$ to $\infty$, except for the endpoint $\xi(t)$ which is required to equal $q(t)$ by the Dirichlet boundary condition. We recognise (3.2) as the standard Feynman path-integral expression for the evolution up to time $t$ of a wavefunction $\psi(q)$ starting out at time $t_{0}$ with the value $\mathcal{O}(q)$. In other words, the operator-state map is nothing other than the application of the evolution operator to the initial wavefunction $\mathcal{O}(q)$, i.e.

$$
\psi_{\mathcal{O}}(\varphi(t))=\lim _{t_{0} \rightarrow-\infty} e^{-\mathrm{i} H_{\mathrm{L}}\left(t-t_{0}\right)} \mathcal{O}\left(\varphi\left(t_{0}\right)\right)
$$

Thus, the operator-state map derives a solution of the Liouville Schrödinger equation from an initial time-asymptotic form of the wavefunction, which may in turn be taken to be of a simple plane-wave form.

The usefulness of this time-asymptotic construction is not a priori obvious in an interacting theory such as the Liouville theory, where the true quantum-mechanical stationary states are quite different from plane waves. In particular, as we saw in section 2 , the true stationary states die off very rapidly under the potential (i.e. at large positive $q$ ), as $\exp \left(-e^{q}-\frac{1}{2} q\right)$, which is quite unlike the oscillatory behaviour of a plane wave state. We shall see, however, that at least in the case of an initial wavefunction $e^{\mathrm{i} p \varphi\left(t_{0}\right)}$ with positive real momentum $p>0$, one does in fact generate a single (un-normalized) Liouville wavefunction of the form (2.2.8). The restriction to real $p>0$ for the success of this construction constitutes the Seiberg bound [4], which is thus seen to be a property of the operator-state map. We shall see that the case with imaginary $p$, which is of importance to noncritical string theory, actually produces a result that does not seem to have been fully taken into account in the string-theory literature. It should be emphasised that our present discussion of the operator-state map does not in any way contradict the multivalued structure of the quantum canonical transformations 
to free fields as discussed in section two. The discussion here concerns the peculiarities of the operator-state map construction, but does not really address the underlying structure of Liouville theory itself. Our purpose in presenting this here in some detail is to compare and contrast the operator-state map to the more intrinsically-motivated canonical transformation between interacting and free theories that we have discussed earlier.

Using the intertwining-operator realisation (2.2.5) of the canonical transformation (2.2.1), one can write $(3.3)$ as

$$
\psi_{\mathcal{O}}(\varphi(t))=\lim _{t_{0} \rightarrow-\infty} C e^{-\mathrm{i} \tilde{H}_{\mathrm{L}}\left(t-t_{0}\right)} C^{-1} \mathcal{O}\left(\varphi\left(t_{0}\right)\right)
$$

Note that in both the free-Hamiltonian picture and in the interacting-Hamiltonian picture, this construction has an ostensible mismatch: from the point of view of (3.3), one is propagating a free-theory wavefunction with the interacting-theory evolution operator. Alternatively, upon noting that the application of $C^{-1}$ to $\mathcal{O}\left(\varphi\left(t_{0}\right)\right)$ gives an interacting-theory wavefunction as in (2.2.2), one sees that the form (3.4) employs a free-theory evolution operator to propagate an interacting-theory wavefunction. Consequently, the only hope for the construction of $(3.3,3.4)$ to give a single Liouville-theory eigenfunction is for just one of the infinity of exact Liouville-theory eigenmodes arising in the decomposition of the initial wavefunction $\mathcal{O}(\varphi)$ to persist in the infinite-time limit, and that all the other modes have amplitudes that decay with time. We now shall see that this hope is realised only in certain cases.

There are two ways of explicitly evaluating the construction (3.4). One can either decompose the initial wavefunction $\mathcal{O}\left(\varphi\left(t_{0}\right)\right)$ into normalized eigenstates $(2.2 .9)$ of the interacting Liouville Hamiltonian and then evolve the latter forward in time by applying their corresponding phase factors and by then recombining, or one can use our construction of the Liouville intertwining operator (2.2.5) to explicitly calculate the Green function for the theory. Both approaches lead to the same final integral expression for the time-dependent wavefunction. Since the latter approach is more in the spirit of our earlier discussion of the Heisenberg-representation time evolution of the quantum operator $e^{-q(t)}(2.2 .19-2.2 .23)$, this is the approach we shall present here.

The Green function for the Liouville Schrödinger equation with a time interval $\Delta t=t-t_{0}$ is given by

$$
G(z, w ; \Delta t)=\left[C^{-1} e^{-\frac{\mathrm{i}}{2} \tilde{p}^{2} \Delta t} C \delta(q-w)\right](z),
$$

where the $q$ variable in $\delta(q-w)$ is transmuted into $z$ by the operator. We begin by evaluating $e^{-\frac{\mathrm{i}}{2} \tilde{p}^{2} \Delta t} C \delta(q-w)$, where $C=\mathcal{P}_{\sinh q} p^{-1} \mathcal{I} \mathcal{P}_{\ln q}$. In applying the first two transformations within $C$ to the delta function, one needs to be careful with the range of integration in the Fourier transform $I$. Specifically, note that the transformation $\mathcal{I P}_{\ln q}$ needs to be the inverse of $\mathcal{P}_{e^{q}} \mathcal{I}^{-1}$, which when acting on functions $f(x)$ is realised by $\left[\mathcal{P}_{e^{q}} \mathcal{I}^{-1} f\right](q)=(2 \pi)^{-\frac{1}{2}} \int_{-\infty}^{\infty} d x e^{-\mathrm{i} x e^{q}} f(x)$. The inverse of this transformation, realised on 
functions $g(u)$ is given by $(2 \pi)^{-\frac{1}{2}} \int_{-\infty}^{\infty} d u e^{u} e^{\mathrm{i} x e^{u}} g(u)$. Writing this in the form $\left[\mathcal{I} \mathcal{P}_{\ln q} g\right](x)$ by making the substitution $q=e^{u}$, one obtains

$$
\left[\mathcal{I P}_{\ln q} g\right](x)=\frac{1}{\sqrt{2 \pi}} \int_{0}^{\infty} d q e^{\mathrm{i} q x} g(\ln q)
$$

The important point to note in (3.6) is that the lower limit of the $q$ integration is 0 and not $-\infty$. Applying (3.6) to the integral representation of the delta function $\delta(q-w)=$ $(2 \pi)^{-1} \int_{-\infty}^{\infty} d k_{1} e^{\mathrm{i} k_{1}(q-w)}$, one obtains

$$
\left[\mathcal{I}_{\ln q} \delta(q-w)\right](x)=\frac{1}{\sqrt{2 \pi}} \int_{0}^{\infty} d q e^{\mathrm{i} q x} \int_{-\infty}^{\infty} \frac{d k_{1}}{2 \pi} e^{-\mathrm{i} k_{1} w} q^{\mathrm{i} k_{1}}
$$

Applying $e^{-\frac{\mathrm{i}}{2} \tilde{p}^{2} \Delta t} \mathcal{P}_{\sinh q} p^{-1}$ to (3.7), one then obtains, using also $p^{-1} \mathcal{I}=\mathcal{I} q^{-1}$,

$$
\begin{aligned}
& {\left[e^{-\frac{\mathrm{i}}{2} \tilde{p}^{2} \Delta t} C \delta(q-w)\right](z)} \\
& =\int_{-\infty}^{\infty} \frac{d k_{2}}{\sqrt{2 \pi}} e^{\mathrm{i} z k_{2}} e^{-\frac{\mathrm{i}}{2} k_{2}^{2} \Delta t} \int_{-\infty}^{\infty} \frac{d x}{\sqrt{2 \pi}} e^{-\mathrm{i} x k_{2}} \int_{-\infty}^{\infty} \frac{d k_{1}}{2 \pi} \int_{0}^{\infty} \frac{d q}{\sqrt{2 \pi}} e^{-\mathrm{i} k_{1} w} q^{\mathrm{i} k_{1}-1} e^{\mathrm{i} q \sinh x} \\
& =\int_{-\infty}^{\infty} d k_{1} \int_{-\infty}^{\infty} \frac{d k_{2}}{(2 \pi)^{\frac{5}{2}}} 2^{\mathrm{i} k_{1}-1} e^{\frac{1}{2} \pi k_{2}} \Gamma\left(\frac{\mathrm{i}\left(k_{1}+k_{2}\right)}{2}\right) \Gamma\left(\frac{\mathrm{i}\left(k_{1}-k_{2}\right)}{2}\right) e^{-\mathrm{i} k_{1} w} e^{\mathrm{i} k_{2} z-\frac{\mathrm{i}}{2} k_{2}^{2} \Delta t}
\end{aligned}
$$

The final stage of applying $C^{-1}$ may then be done using

$$
C^{-1} e^{\mathrm{i} k_{2} z}=\frac{2 k_{2}}{\sqrt{2 \pi}} e^{\frac{\pi}{2} k_{2}} K_{\mathrm{i} k_{2}}\left(e^{z}\right)
$$

as derived earlier in (2.2.8). The final result for the Green function is

$$
\begin{aligned}
& G(z, w ; \Delta t)= \\
& \int_{-\infty}^{\infty} d k_{1} \int_{-\infty}^{\infty} \frac{d k_{2}}{(2 \pi)^{3}} 2^{\mathrm{i} k_{1}} k_{2} e^{\pi k_{2}} K_{\mathrm{i} k_{2}}\left(e^{z}\right) \Gamma\left(\frac{\mathrm{i}\left(k_{1}+k_{2}\right)}{2}\right) \Gamma\left(\frac{\mathrm{i}\left(k_{1}-k_{2}\right)}{2}\right) e^{-\mathrm{i} k_{1} w} e^{-\frac{\mathrm{i}}{2} k_{2}^{2} \Delta t} .
\end{aligned}
$$

Applying $\int_{-\infty}^{\infty} d w G(q, w ; \Delta t)$ to an initial plane-wave wavefunction at time $t_{0}$

$$
\left.\psi\right|_{\mathcal{O}_{p}}\left(w, t_{0}\right)=e^{\mathrm{i} p w}
$$

one obtains the time-evolved wavefunction

$$
\left.\psi\right|_{\mathcal{O}_{p}}(q, t)=2^{\mathrm{i} p} \int_{-\infty}^{\infty} \frac{d k}{(2 \pi)^{2}} k e^{\pi k} K_{\mathrm{i} k}\left(e^{q}\right) \Gamma\left(\frac{\mathrm{i}(p+k)}{2}\right) \Gamma\left(\frac{\mathrm{i}(p-k)}{2}\right) e^{-\frac{\mathrm{i}}{2} k^{2} \Delta t} .
$$

The behaviour of $\left.\psi\right|_{\mathcal{O}_{p}}(q, t)$ as $\Delta t \rightarrow \infty$ may be evaluated by contour-integral methods. In doing this, the point to note is that for $k=e^{-\mathrm{i} \pi / 4} k_{m}$, the factor $e^{-\mathrm{i} k^{2} \Delta t / 2}$ becomes a 
representation of a delta function, $e^{-k_{m}^{2} \Delta t / 2} \rightarrow \sqrt{(2 \pi / \Delta t)} \delta\left(k_{m}\right)$. This causes the contributions to an integral of the form (3.12), but rotated to the diagonal contour $\operatorname{Im} k=-\operatorname{Re} k$, to become concentrated near the origin where the integrand vanishes, owing to the factor of $k_{m}$. The rotated integral may be evaluated by steepest descents using

$$
\begin{aligned}
\int_{-\infty}^{\infty} d k_{m} k_{m} f\left(k_{m}\right) e^{-\frac{1}{2} k_{m}^{2} \Delta t} & =\frac{1}{\Delta t} \int_{-\infty}^{\infty} d k_{m} f^{\prime}\left(k_{m}\right) e^{-\frac{1}{2} k_{m}^{2} \Delta t} \\
& \rightarrow \sqrt{2 \pi}(\Delta t)^{-\frac{3}{2}} f^{\prime}(0),
\end{aligned}
$$

with $f\left(k_{m}\right)=\exp \left(\pi e^{-\mathrm{i} \pi / 4} k_{m}\right) K_{e^{\mathrm{i} \pi / 4} k_{m}}\left(e^{q}\right) \Gamma\left(\mathrm{i}\left(p+e^{-\mathrm{i} \pi / 4} k_{m}\right) / 2\right) \Gamma\left(\mathrm{i}\left(p-e^{-\mathrm{i} \pi / 4} k_{m}\right) / 2\right)$. Noting that this expression is $\exp \left(\pi e^{-\mathrm{i} \pi / 4} k_{m}\right)$ times a factor even in $k_{m}$, we obtain the large-time limit for the rotated integral

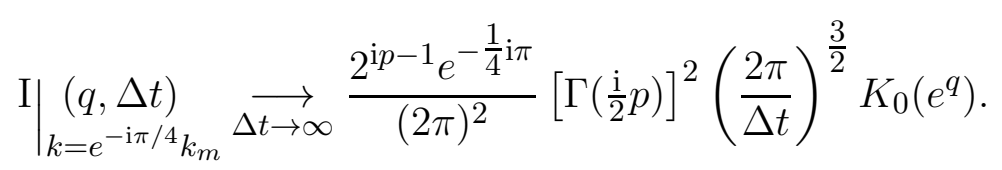

Thus, the rotated integral falls off as $(\Delta t)^{-3 / 2}$ for large $\Delta t$. Consequently, the value of $\left.\psi\right|_{\mathcal{O}_{p}}(q, t)$ in the limit of large $\Delta t$ is given entirely by contributions from poles of the integrand that become enclosed when joining the original integral (3.12) to the rotated integral. These integrals are joined by two octant arcs at infinity, $(3 \pi / 4)<\theta<\pi$ and $(7 \pi / 4)<\theta<2 \pi$, on which the $e^{-k^{2} \Delta t / 2}$ factor causes the arc integrals to vanish.

The determination of the specific poles of the integrand that become enclosed by the contour depends on the value of the momentum $p$ of the initial wavefunction (3.11). The poles in (3.12) arise only from the $\Gamma$ functions, since the rest of the integrand is an entire function of $k$. The Gamma function $\Gamma\left(\frac{\mathrm{i}(p+k)}{2}\right)$ has poles at $k=-p+2 \mathrm{i} n$, while the Gamma function $\Gamma\left(\frac{\mathrm{i}(p-k)}{2}\right)$ has poles at $k=p-2 \mathrm{i} n$, where $n \in Z, n \geq 0$.

The easiest case to discuss (which we shall denote (I)) is that of imaginary momentum $p=-\mathrm{i} \beta$, with $\beta>0$, for which the initial wavefunction takes the form

$$
\left.\psi\right|_{\mathcal{O}_{-\mathrm{i} \beta}}\left(q, t_{0}\right)=e^{\beta q}
$$

As can be seen from Figure 1, no poles are enclosed by the contour in this case. Consequently, the integral (3.12) is given entirely by (3.14), and falls off like $(\Delta t)^{-3 / 2}$ in this case.

Thus when the momentum $p$ of the initial wavefunction is given by $p=-\mathrm{i} \beta$ with $\beta>0$, there is no persistent Liouville eigenfunction at late times. This property has been expressed in the literature by the statement that "vertex operators $e^{\alpha \phi}$ with $\alpha<-Q$ do not exist" $[5] . \ddagger$ The bound $\beta<0$ on the imaginary momenta of initial wavefunctions that can leave

$\ddagger$ Restated in the conventions of this paper. Recall that the momentum of the vertex operator $e^{\alpha \phi}$ is shifted by the background charge $Q$ relative to the momentum of the initial wavefunction, and so $\alpha=\frac{\gamma}{2} \beta-Q$, where $\gamma$ is a negative multiple of $Q$ as given in (1.3); note also that $\varphi$ and $q$ are related as in (2.1a). 
persistent Liouville eigenfunctions at late times is known as the "Seiberg bound" [4]. Case (I), where there is no persistent eigenfunction, is known as the "anti-Seiberg" case.



Figure 1: Integration contour for the operator-state map with imaginarymomentum $p=-\mathrm{i} \beta$, anti-Seiberg case $(\beta>0)$.

The other cases of interest may be treated by analytic continuation in $p$ from case (I), for which the integral in (3.12) is the most clearly convergent. In making such analytic continuations, it is important not to let a pole actually touch the integration contour. Thus, in order to treat the cases of real momentum $p$, it is necessary to rotate $p$ from the negative imaginary axis to an infinitesimal amount $\epsilon$ short of the real axis, rotating either counterclockwise to obtain case (II): $p$ real, $p>0$, or clockwise to obtain case (III): $p$ real, $p<0$. These cases are shown in Figure 2.

As can be seen, in case (II), $p>0$, the $n=0$ Gamma function pole at $k=-p$ is 
enclosed, as well as a number $§$ of $n>0$ resonance poles, depending on the value of $p$. Since these poles have a time dependence with amplitude $e^{-2 n p \Delta t}$, only the $n=0$ pole survives in the large $\Delta t$ limit. Thus, for real $p>0$, the time-evolved wavefunction (3.12) does in fact settle down to become a single (un-normalized) Liouville eigenfunction $K_{\mathrm{i} p}\left(e^{q}\right)$ (once again, a single wavefunction occurs because $K_{\mathrm{i} p}$ is symmetric in $p$ ).

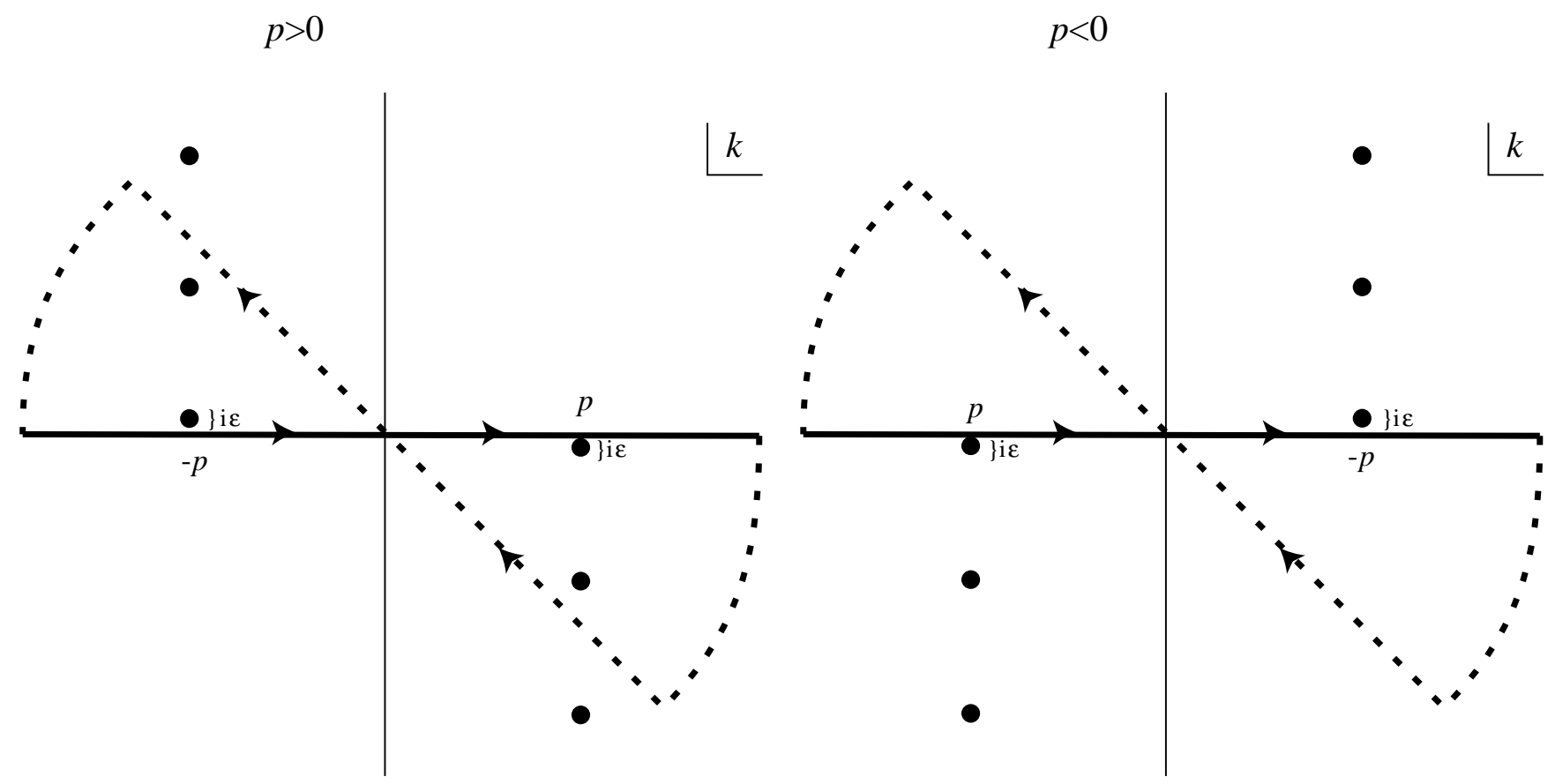

Figure 2: Integration contours for the operator-state map with realmomentum.

The clockwise rotation from case (I) to case (III) leaves all Gamma-function poles lying outside the integration contour. Note that the requirement of stopping the rotation just $\epsilon$ short of bringing $p$ onto the real axis, in order to preserve convergence means that even the $n=0$ pole is left outside the contour. Consequently, in case (III), of real $p<0$, the only contribution to (3.12) in the large $\Delta t$ limit is just (3.14), decaying like $(\Delta t)^{-3 / 2}$.

The results for cases (II) and (III) taken together show that, starting from an initial wavefunction with real momentum $p$, one only obtains a persisting Liouville eigenfunction at late times if $p$ is positive. The time-asymptotic nature of the operator-state map may be used to give a heuristic picture of the difference between the real-momentum cases (II) and (III). In case (II), if one starts from an initial wavefunction with momentum directed toward the Liouville potential "mountain", the incoming right-moving wave is reflected as time goes on, producing a left-moving wave. At the same time, the wavefunction under the potential decays, contributing to the left-moving wave. In the large $\Delta t$ limit, the right-moving and left-moving waves combine to form a standing-wave eigenfunction $\psi_{p}(q)$. In case (III), on

\footnotetext{
$\S$ The number of poles enclosed is $2\left[\frac{1}{2} p\right]+2$, where $[x]$ is the integer part of the real number $x$.
} 
the other hand, one starts with a left-moving wave. As time goes on, the wavefunction under the potential decays, contributing more to the left-moving wave. In this case, however, there is no reflection and hence no contribution to any right-moving wave. Thus, there is no way to produce a single standing-wave eigenfunction. As a result, the wavefunction at all finite values of $q$ decays like $(\Delta t)^{-3 / 2}$.

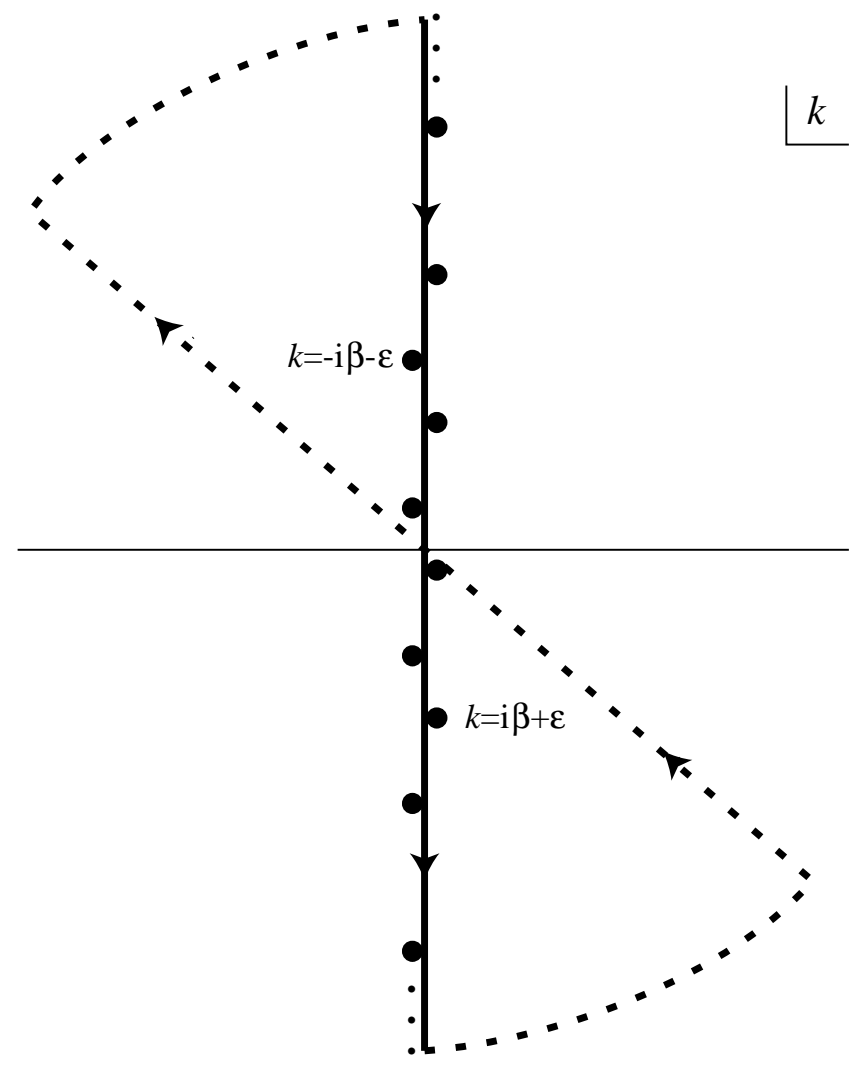

Figure 3: Integration contour for the operator-state map with imaginarymomentum $p=-\mathrm{i} \beta$, Seiberg case $(\beta<0)$.

The final case (IV), of imaginary $p=-\mathrm{i} \beta, \beta<0$, has been called the "Seiberg" case. It is not possible to reach this configuration of the poles directly by starting from case (I) without having some poles cross the real-axis contour of the original integral (3.12). However, one may define this case by a two-step procedure of analytic continuation. First, analytically continue $p=-\mathrm{i} \beta$ in case (I) so as to give $p$ an infinitesimal negative real part $\epsilon$. Next, rotate the contour of the integral (3.12) so as to make it run along the imaginary axis, by adding quadrant arcs $\pi / 2<\theta<\pi$ and $(3 \pi) / 2<\theta<2 \pi$, along which the integral vanishes. The shifted poles and the rotated contour are shown in Figure 3. At this stage, one can now analytically continue in $p$ parallel to the imaginary $k$ axis without having poles cross the new contour. Evaluation of this redefined form of the original integral (3.12) then proceeds as in cases (I), (II) and (III), adding octant arcs with vanishing contributions and also the diagonal 
integral along the contour $\operatorname{Im} k=-\operatorname{Re} k$, which again gives a decaying contribution (3.14). As can be seen, a number of the Gamma-function poles are now enclosed by this contour. Moreover, unlike in case (II), all of these poles now have a pure-phase time evolution, since $\left(-\mathrm{i} k^{2} \Delta t\right)$ is purely imaginary (up to infinitesimal terms from the regularization) for poles lying along the imaginary axis. Thus, in addition to the expected Liouville eigenfunction $K_{-\beta}\left(e^{q}\right)$, one obtains also a whole chorus of resonant eigenfunctions $\Phi K_{-\beta-2 n}\left(e^{q}\right)$ with imaginary momenta $\mathrm{i} k_{\mathrm{m}}$ extending through positive values from $k_{\mathrm{m}}=-\beta$ down to zero, at intervals $\Delta k_{\mathrm{m}}=2$.

In the imaginary-momentum cases $p=-\mathrm{i} \beta$ (I, IV) that are relevant in non-critical string theory, we have confirmed the absence of persistent eigenfunctions from the operator-state map construction as expected in the anti-Seiberg case $\beta>0$, but we get more than we bargained for in the Seiberg case $\beta<0$ : we find a momentum-dependent number of modes persisting in the limit $\Delta t \rightarrow \infty$.

It is useful at this stage to summarize how the four cases (I-IV) relate to one another. The integral (3.12) is most clearly defined in the anti-Seiberg case (I). From this case, the others have been obtained by analytic continuation in the momentum $p$, and also, for case (IV), in the defining contour of the integral. One may visualize the lines of poles of the Gamma functions in (3.12) as a "stalagmite" rising from the floor with its tip located at $k=p$, and a "stalactite" descending from the ceiling with its tip located at $k=-p$. In cases (I), (II) and (III), these spikes do not pierce the real $k$ axis, which forms the boundary of the region of convergence of (3.12) as originally defined. In these three cases, one obtains a nonvanishing result at large times whenever there are poles enclosed by the octant wedges of the closed contour of integration, as shown in Figs 1 and 2. In case (IV), where $p=-\mathrm{i} \beta$ with $\beta<0$, the stalagmite and stalactite have pierced the real $k$ axis, as shown in Fig. 3. In consequence, one must be careful here in performing an analytic continuation from any of the cases (I) (II) or (III). In particular, a consistent analytic continuation to this case can be achieved by deforming the defining contour of the integral (3.12) into one running along the imaginary axis; this procedure avoids ever having a pole actually touch the defining contour of integration. Note futher that the only cases in which (3.12) can asymptotically settle down to have a time-independent amplitude arise when some of the poles lie infinitesimally close to either the real or imaginary $k$ axes.

We conclude this section by comparing our findings on the operator-state map with previous discussions in the literature. When the momentum is imaginary, $k=\mathrm{i} k_{\mathrm{I}}$, the asymptotic form (2.2.18) of the Liouville eigenfunctions at large negative $q$ (i.e. away from the potential) diverges exponentially, regardless of the sign of $k_{\mathrm{I}}$. Thus (paraphrasing in the language of this paper) it was argued [31] that if an initial wavefunction $e^{\beta q}$ had $\beta>0$, it could not give rise to a persisting Liouville eigenfunction at late times. Our results for case (I) agree with this assertion. As far as we are aware, however, it has generally been assumed

I The number of distinct persisting Liouville eigenfunctions is $\left[-\frac{1}{2} \beta\right]+1$. Recall that $\beta$ is negative here. 
that in case (IV), where $\beta<0$, an initial wavefunction $e^{\beta q}$ would result in a single persisting Liouville eigenfunction $K_{-\beta}\left(e^{q}\right)$. Our results show that this is not generally what happens, and instead one gets a number of Liouville eigenfunctions $K_{-\beta-2 n}\left(e^{q}\right)$, with $n=0,1,2$, $\ldots\left[-\frac{1}{2} \beta\right]$. In view of this finding, it is no longer clear that the use of exponential vertex operators, which is very natural in a free-field theory, is so appropriate in Liouville theory.

If one wants to make use of results from free-field theory in order to study the dynamics of Liouville theory, it is more appropriate to use the canonical transformation as implemented by intertwining operators, as we have discussed in section two. In contrast to the operator-state map, which is a time-asymptotic construction that aims to obtain Liouville eigenfunctions from an initial plane-wave form of the wavefunction, the intertwining-operator construction may be thought of as deriving the Liouville wavefunctions from spatially-asymptotic limits. One may view the effect of the canonical transformation this way because the exact wavefunctions $\psi_{p}(q)$ asymptotically approach linear combinations of free-field exponentials $e^{ \pm i p q}$ as $q \rightarrow-\infty$. As we have seen, this spatially-asymptotic identification holds only up to Weylgroup transformations: the two-to-one nature of the canonical transformation is expressed by the fact that both $e^{\mathrm{i} p \tilde{q}}$ and $e^{-\mathrm{i} p \tilde{q}}$ are transformed to $\psi_{p}(q)$.

\section{Canonical transformations for Toda gravity}

For the minimal (two-scalar) non-critical $W_{3}$ string, we derived the allowable forms of the potentials in Eqs (1.8). It will now be convenient to make a field redefinition in order to bring the resulting minisuperspace Toda Lagrangian to a standard form. Accordingly, we set

$$
\begin{aligned}
\varphi_{1}(\tau, \sigma) & =-\frac{4 Q_{1}}{7} q_{1}(\tau)+\varphi_{1}^{\mathrm{osc}}(\tau, \sigma) \\
\varphi_{2}(\tau, \sigma) & =-\frac{4 Q_{2}}{7}\left(2 q_{2}(\tau)-q_{1}(\tau)\right)+\varphi_{2}^{\mathrm{osc}}(\tau, \sigma), \\
\oint d \sigma \varphi_{1}^{\mathrm{osc}} & =\oint d \sigma \varphi_{2}^{\mathrm{osc}}=0 .
\end{aligned}
$$

The action for the minisuperspace modes $q_{1}$ and $q_{2}$ in this basis becomes, again denoting the time parameter for the quantum-mechanical system by $t$,

$$
I_{\mathrm{T}}=\int d t\left(\dot{q}_{1}^{2}+\dot{q}_{2}^{2}-\dot{q}_{1} \dot{q}_{2}-e^{2 q_{1}-q_{2}}-e^{2 q_{2}-q_{1}}\right),
$$

which can be recognised as the action for Toda mechanics in the Chevalley basis for $A_{2} \equiv$ $S U(3)$. The Hamiltonian following from (4.2) is then

$$
H_{\mathrm{T}}=\frac{1}{3}\left(p_{1}^{2}+p_{2}^{2}+p_{1} p_{2}\right)+e^{2 q_{1}-q_{2}}+e^{2 q_{2}-q_{1}},
$$


giving equations of motion

$$
\begin{array}{ll}
p_{1}=2 \dot{q}_{1}-\dot{q}_{2} & p_{2}=2 \dot{q}_{2}-\dot{q}_{1} \\
\ddot{q}_{1}=-e^{2 q_{1}-q_{2}} & \ddot{q}_{2}=-e^{2 q_{2}-q_{1}} .
\end{array}
$$

\subsection{Classical Toda Mechanics}

Proceeding in analogy to our Liouville discussion of section 2, we first seek solutions to the classical Toda mechanics equations (4.4), following the style of Ref. [35], starting from the ansatz

$$
e^{-q_{1}}=\sum_{i=1}^{3} f_{i} e^{\mu_{i} t} \quad e^{-q_{2}}=\sum_{i=1}^{3} g_{i} e^{-\mu_{i} t},
$$

where $\mu_{i}, f_{i}$ and $g_{i}$ are constants. Substituting into (4.4b), we find that one must require

$$
\begin{array}{r}
\mu_{1}+\mu_{2}+\mu_{3}=0 \\
f_{1} f_{2} f_{3}\left(\mu_{1}-\mu_{2}\right)^{2}\left(\mu_{2}-\mu_{3}\right)^{2}\left(\mu_{3}-\mu_{1}\right)^{2}=1 \\
g_{1}=f_{2} f_{3}\left(\mu_{2}-\mu_{3}\right)^{2}, \quad \text { and cyclically. }
\end{array}
$$

Solutions of this form, which have four free parameters after taking Eqs (4.1.2) into account, are in fact general solutions to the classical equations of motion (4.4).

It is convenient to write $\mu_{1} t=\tilde{q}_{1}$ and $\mu_{2} t=\tilde{q}_{2}$ and to reparametrise the general solution (4.1.1) subject to (4.1.2), as $f_{1}=g_{1}=\left[\tilde{p}_{1}\left(\tilde{p}_{1}-\tilde{p}_{2}\right)\right]^{-1}, f_{2}=g_{2}=\left[\tilde{p}_{2}\left(\tilde{p}_{1}-\tilde{p}_{2}\right)\right]^{-1}, f_{3}=g_{3}=$ $\left[\tilde{p}_{1} \tilde{p}_{2}\right]^{-1}$. The solution to (4.4) then takes the form

$$
\begin{aligned}
e^{-q_{1}} & =\frac{1}{\tilde{p}_{1}\left(\tilde{p}_{1}-\tilde{p}_{2}\right)} e^{\tilde{q}_{1}}+\frac{1}{\tilde{p}_{2}\left(\tilde{p}_{1}-\tilde{p}_{2}\right)} e^{\tilde{q}_{2}}+\frac{1}{\tilde{p}_{1} \tilde{p}_{2}} e^{-\tilde{q}_{1}-\tilde{q}_{2}} \\
e^{-q_{2}} & =\frac{1}{\tilde{p}_{1}\left(\tilde{p}_{1}-\tilde{p}_{2}\right)} e^{-\tilde{q}_{1}}+\frac{1}{\tilde{p}_{2}\left(\tilde{p}_{1}-\tilde{p}_{2}\right)} e^{-\tilde{q}_{2}}+\frac{1}{\tilde{p}_{1} \tilde{p}_{2}} e^{\tilde{q}_{1}+\tilde{q}_{2}} \\
\left(2 p_{1}+p_{2}\right) e^{-q_{1}} & =-\frac{\left(2 \tilde{p}_{1}-\tilde{p}_{2}\right)}{\tilde{p}_{1}\left(\tilde{p}_{1}-\tilde{p}_{2}\right)} e^{\tilde{q}_{1}}-\frac{\left(2 \tilde{p}_{2}-\tilde{p}_{1}\right)}{\tilde{p}_{2}\left(\tilde{p}_{1}-\tilde{p}_{2}\right)} e^{\tilde{q}_{2}}+\frac{\left(\tilde{p}_{1}+\tilde{p}_{2}\right)}{\tilde{p}_{1} \tilde{p}_{2}} e^{-\tilde{q}_{1}-\tilde{q}_{2}} \\
\left(2 p_{2}+p_{1}\right) e^{-q_{2}} & =\frac{\left(2 \tilde{p}_{1}-\tilde{p}_{2}\right)}{\tilde{p}_{1}\left(\tilde{p}_{1}-\tilde{p}_{2}\right)} e^{-\tilde{q}_{1}}+\frac{\left(2 \tilde{p}_{2}-\tilde{p}_{1}\right)}{\tilde{p}_{2}\left(\tilde{p}_{1}-\tilde{p}_{2}\right)} e^{-\tilde{q}_{2}}-\frac{\left(\tilde{p}_{1}+\tilde{p}_{2}\right)}{\tilde{p}_{1} \tilde{p}_{2}} e^{\tilde{q}_{1}+\tilde{q}_{2}} .
\end{aligned}
$$

The solution (4.1.3) may be interpreted as a canonical transformation $F$ from the interacting Toda-theory variables $\left(q_{1}, q_{2} ; p_{1}, p_{2}\right)$ to free variables $\left(\tilde{q}_{1}, \tilde{q}_{2} ; \tilde{p}_{1}, \tilde{p}_{2}\right)$. One may verify directly that this transformation is indeed canonical. The Toda Hamiltonian (4.3) maps into the free Hamiltonian*

$$
\tilde{H}_{\mathrm{T}}=\frac{1}{3}\left(\tilde{p}_{1}^{2}+\tilde{p}_{2}^{2}-\tilde{p}_{1} \tilde{p}_{2}\right)
$$

* Note that one could bring the the free Hamiltonian into the same form as the kinetic part of the interacting Hamiltonian (4.3), e.g. by tacking onto (4.1.3) an additional canonical transformation $\left(p_{1}, q_{1}\right) \mapsto$ $\left(-\tilde{p}_{1},-\tilde{q}_{1}\right)$. We shall not do so here, but shall need to include such an additional transformation later on for comparison with results derived in the literature for the free-field $W_{3}$ string, without the interaction potentials (1.8). 
Note that this implies that the free momenta $\tilde{p}_{1}$ and $\tilde{p}_{2}$ are related to the free velocities by

$$
\tilde{p}_{1}=2 \dot{\tilde{q}}_{1}+\dot{\tilde{q}}_{2} \quad \tilde{p}_{2}=2 \dot{\tilde{q}}_{2}+\dot{\tilde{q}}_{1}
$$

The exact classical integrability of the Toda system has another aspect, namely the existence of a second independent constant of the motion. This is of course just the minisuperspace limit of the spin-3 current (1.6) of the $W_{3}$ string, after taking into account appropriate shifts of the momenta by background charges as in (2.3). For the interacting theory (4.2), this second invariant is

$$
\begin{aligned}
W_{\mathrm{T}} & =\frac{1}{9} p_{1}^{3}+\frac{1}{6} p_{1}^{2} p_{2}-\frac{1}{6} p_{1} p_{2}^{2}-\frac{1}{9} p_{2}^{3}+\frac{1}{2}\left(2 p_{2}+p_{1}\right) e^{2 q_{1}-q_{2}}-\frac{1}{2}\left(2 p_{1}+p_{2}\right) e^{2 q_{2}-q_{1}} \\
& =\frac{1}{18}\left(2 p_{1}+p_{2}\right)\left(2 p_{2}+p_{1}\right)\left(p_{1}-p_{2}\right)+\frac{1}{2}\left(2 p_{2}+p_{1}\right) e^{2 q_{1}-q_{2}}-\frac{1}{2}\left(2 p_{1}+p_{2}\right) e^{2 q_{2}-q_{1}} .
\end{aligned}
$$

Upon use of the canonical transformation $F(4.1 .3)$, this becomes a purely cubic invariant of the free theory,

$$
\begin{aligned}
\tilde{W}_{\mathrm{T}} & =-\frac{1}{9} \tilde{p}_{1}^{3}+\frac{1}{6} \tilde{p}_{1}^{2} \tilde{p}_{2}+\frac{1}{6} \tilde{p}_{1} \tilde{p}_{2}^{2}-\frac{1}{9} \tilde{p}_{2}^{3} \\
& =\frac{1}{18}\left(2 \tilde{p}_{1}-\tilde{p}_{2}\right)\left(2 \tilde{p}_{2}-\tilde{p}_{1}\right)\left(\tilde{p}_{1}+\tilde{p}_{2}\right) .
\end{aligned}
$$

The two invariants $\tilde{H}_{\mathrm{T}}$ and $\tilde{W}_{\mathrm{T}}$ determine the discrete symmetries of the free theory obtained from the Toda theory by our canonical transformation (4.1.3). These discrete symmetries in turn determine the multiplet of forms of the canonical transformation, in analogy to $(2.1 .3),(2.1 .7)$. Requiring that (4.1.4) and (4.1.7) be symmetric determines the following group of transformations. Firstly, the free theory has a threefold symmetry under rotations of the free momenta $\tilde{p}_{1}$ and $\tilde{p}_{2}$ accompanied by corresponding rotations of the free variables $\tilde{q}_{1}$ and $\tilde{q}_{2} ;$ these are generated by

$$
M: \quad\left(\tilde{q}_{1}, \tilde{q}_{2} ; \tilde{p}_{1}, \tilde{p}_{2}\right) \rightarrow\left(-\tilde{q}_{1}-\tilde{q}_{2}, \tilde{q}_{1} ;-\tilde{p}_{2}, \tilde{p}_{1}-\tilde{p}_{2}\right)
$$

The full threefold symmetry is obtained by acting with $M^{0}, M^{1}$ or $M^{2}$. In addition, there is a twofold reflection symmetry,

$$
R: \quad\left(\tilde{q}_{1}, \tilde{q}_{2} ; \tilde{p}_{1}, \tilde{p}_{2}\right) \rightarrow\left(\tilde{q}_{2}, \tilde{q}_{1} ; \tilde{p}_{2}, \tilde{p}_{1}\right)
$$

so that the full discrete symmetry group for the free theory as determined by the invariants (4.1.4), (4.1.7) is $S_{3}$, the permutation group of three objects. As foreshadowed by our Liouville discussion in section two, this is precisely the Weyl group of $A_{2} \equiv S U(3)$, the underlying algebra of the Toda theory.

Another important analogue of the Liouville case is the fact that the classical canonical transformation $F(4.1 .3)$ has only a limited domain of applicability in the $\left(\tilde{p}_{1}, \tilde{p}_{2}\right)$ plane, since the left-hand sides of $(4.1 .3 a, b)$ are always positive. Other domains in the free-momentum plane are obtained by applying the Weyl-group transformations (4.1.8), (4.1.9) to the freetheory variables in $F$, generating a corresponding Weyl multiplet of forms of the canonical 
transformation. We start from a reference region in the free-momentum plane, in which $(4.1 .3 a, b)$ are clearly applicable:

$$
\tilde{p}_{2}>0 ; \quad \tilde{p}_{1}>\tilde{p}_{2},
$$

which is in fact the principal Weyl chamber of $S U(3)$ in the Chevalley basis. Note that in this basis, the Weyl chambers occupy $45^{\circ}$ and $90^{\circ}$ wedges rather than the uniform $60^{\circ}$ wedges of an orthonormal basis.

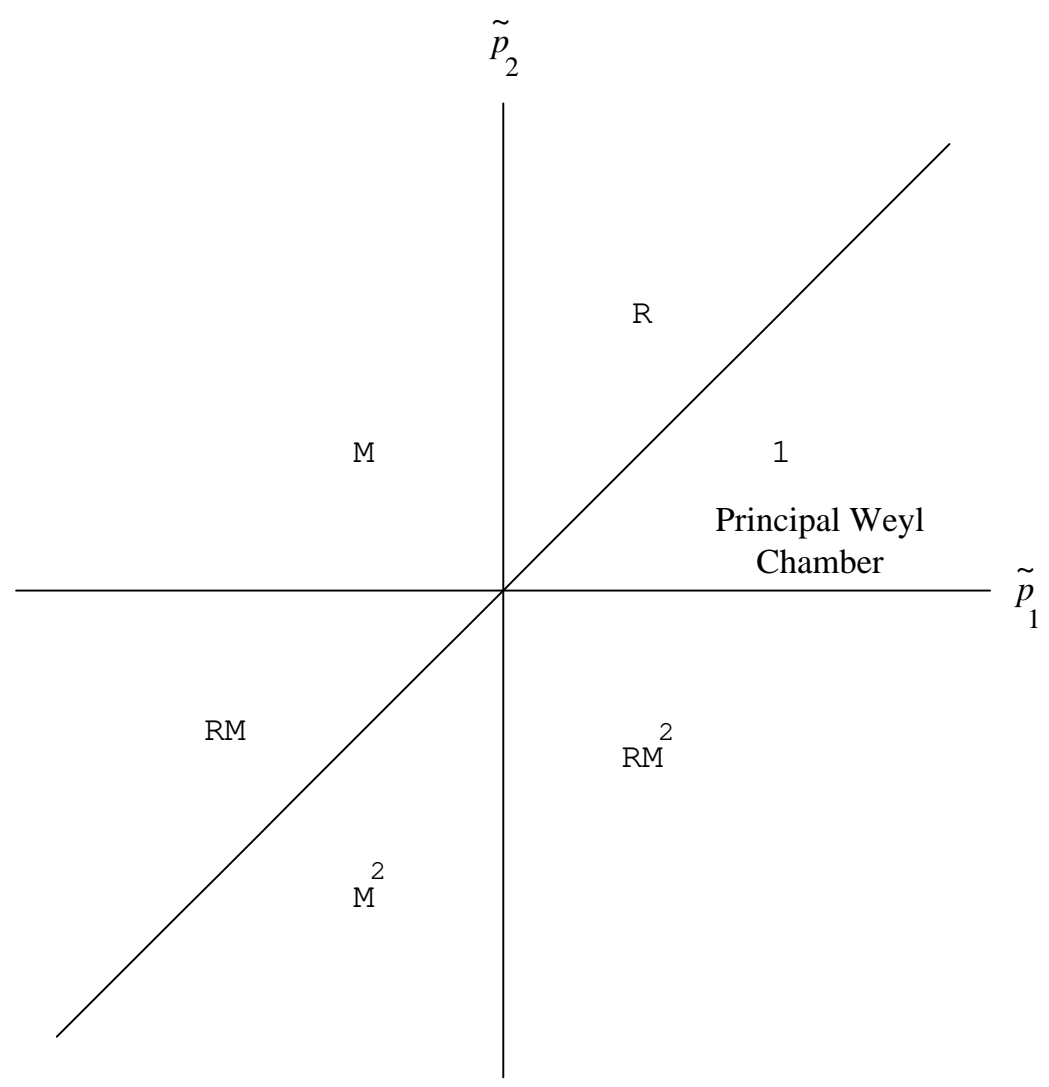

Figure 4: The Weyl chambers. Images of the point labelled "1" in the principalchamber are shown by the corresponding Weyl-group elements.

It is easy to check that, by acting with $M$ zero, one or two times and with $R$ zero or one time, one generates six non-overlapping regions whose union covers the entire free-momentum plane, as shown in Figure 4. In order to link these domains with the corresponding forms of the canonical transformation, it is necessary to recognise another feature of the transformation: there is in fact only a triplet of distinctly different maps obtained from $F$ by application of the Weyl group, because (4.1.3) is invariant under the order-two transformation

$$
Z: \quad\left(\tilde{q}_{1}, \tilde{q}_{2} ; \tilde{p}_{1}, \tilde{p}_{2}\right) \rightarrow\left(-\tilde{q}_{1}-\tilde{q}_{2}, \tilde{q}_{2} ;-\tilde{p}_{1},-\tilde{p}_{1}+\tilde{p}_{2}\right)
$$

which is the Weyl group element $R M$. Acting with this transformation on the reference 
region (4.1.10) generates the region

$$
\tilde{p}_{2}<0 ; \quad \tilde{p}_{1}<\tilde{p}_{2}
$$

obtained from the former by inversion through the origin of the free-momentum plane. Eqs $(4.1 .3 a, b)$ are once again clearly applicable in the region (4.1.12). Owing to the invariance (4.1.11), we have, even for the starting transformation $F$, a situation similar to that of (2.1.7) in the Liouville case: the inverse of (4.1.3) has a twofold branch structure, because one has to choose a set of free momenta either from the region (4.1.10) or from the region (4.1.12). Thus, the fundamental reference region within which (4.1.3) has a uniquely-defined inverse may be taken to be the principal Weyl chamber (4.1.10). Upon application of the rotations $M^{0}, M^{1}, M^{2}$ to the pair of regions $(4.1 .10,4.1 .12)$, one then completely covers the free-momentum plane in a non-overlapping fashion; the three images of this pair are in one-to-one correspondence with the distinct transformations $F, M F, M^{2} F$.

In sum, application of the Weyl group $S_{3}$ to our starting canonical transformation $F$ (4.1.3) and to its principal Weyl-chamber region (4.1.10) produce three distinct forms of the transformation and six distinct Weyl chambers. Considering the union of these regions together with their respective forms of the transformation as a single canonical-transformation map, we see that one has a six-fold branch structure in the $A_{2}$ Toda case, giving a six-to-one relation between free-field solutions and the interacting Toda-theory solutions.

As earlier in the Liouville case, it is instructive to examine the asymptotic relationship between the free-field momenta and the interacting Toda-theory momenta for solutions to the classical equations of motion (4.4). Consider the transformation $F$ (4.1.3) for free momenta in the principal Weyl chamber (4.1.10) in the limit as $t \rightarrow-\infty$. In (4.1.3a), the dominant term on the right-hand side in this limit is $e^{-\left(\tilde{q}_{1}+\tilde{q}_{2}\right)}=e^{-\frac{1}{3}\left(\tilde{p}_{1}+\tilde{p}_{2}\right) t}$, using relation $(4.1 .5)$ between the free momenta and velocities. Thus, in this limit, we have $e^{-q_{1}} \rightarrow\left(\tilde{p}_{1} \tilde{p}_{2}\right)^{-1} e^{-\left(\tilde{q}_{1}+\tilde{q}_{2}\right)}$. Similarly, in $(4.1 .3 b)$, the dominant term in the $t \rightarrow-\infty$ limit is $e^{-\tilde{q}_{1}}=e^{-\frac{1}{3}\left(2 \tilde{p}_{1}-\tilde{p}_{2}\right) t}$, so we obtain $e^{-q_{2}} \rightarrow\left[\tilde{p}_{1}\left(\tilde{p}_{1}-\tilde{p}_{2}\right)\right]^{-1} e^{-\tilde{q}_{1}}$. Then, from $(4.1 .3 c, d)$ we obtain the asymptotic relations between Toda and free momenta: $p_{1} \rightarrow \tilde{p}_{2} ; p_{2} \rightarrow \tilde{p}_{1}-\tilde{p}_{2}$. Although the details of the asymptotic relations change as one goes through the six different Weyl chambers, the pattern that we found in our Liouville discussion is repeated here: Weyl-group images of the starting free-field momenta $\left(\tilde{p}_{1}, \tilde{p}_{2}\right)$ all describe one and the same Toda solution. Thus, we may now concentrate just on the transformations (4.1.3) for the Weyl chamber (4.1.10).

The region of classically-realisable asymptotic Toda momenta for $t \rightarrow-\infty$ following from the above discussion is the whole first quadrant of the Toda-momentum plane: $p_{1}>0$, $p_{2}>0$. The Toda velocities obtained from $(4.1 .5)$ are $\dot{q}_{1}=\frac{1}{3}\left(2 p_{1}+p_{2}\right), \dot{q}_{2}=\frac{1}{3}\left(2 p_{2}+p_{1}\right)$. Thus, the classically-realisable velocities for $t \rightarrow-\infty$ must lie in the wedge $2 \dot{q}_{2}-\dot{q}_{1}>0$, $2 \dot{q}_{1}-\dot{q}_{2}>0$. This wedge corresponds precisely to the asymptotic directions of approach allowed by the "valley" in the Toda potential, as shown in Figure 5. Unlike the Liouville 
case, where by dimensionality the motion is constrained to aim straight at the "mountain", the Toda case in one dimension higher affords a trajectory of motion aimed at the "ravine," or mildest ascent, in the direction opposite to the valley.

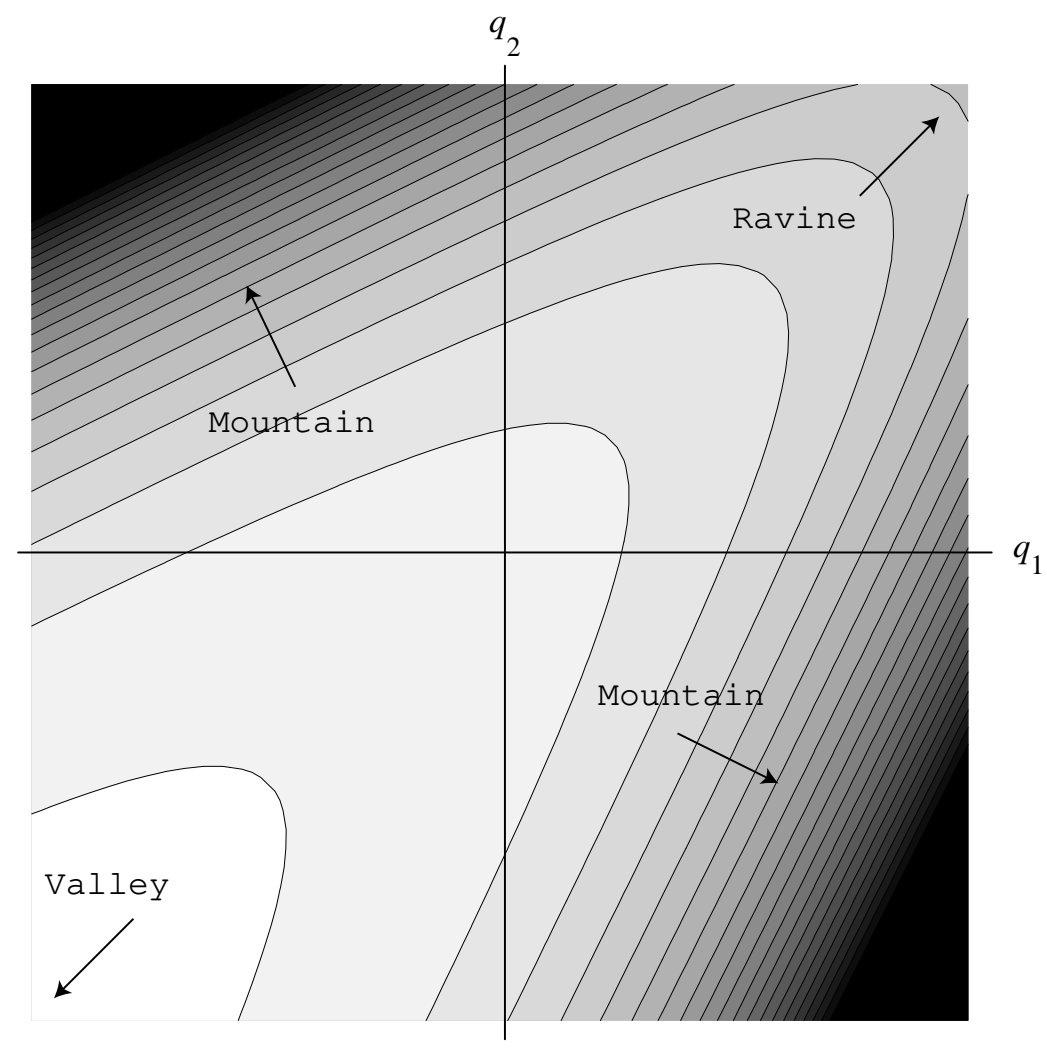

Figure 5: Contour plot of the Toda potential. Darkening gray levels indicaterising values; arrows point up the "mountains" and "ravine," and down the "valley." Classical motionbegins and ends in the valley, with directions of initial and final velocites bounded by theasymptotes to the contour lines.

One may make an analogous asymptotic analysis of the motion for $t \rightarrow+\infty$, considering again only the free solutions corresponding to (4.1.3), (4.1.10). In this case, for $e^{-q_{1}}$ the dominant term is $e^{\tilde{q}_{1}}$ and for $e^{-q_{2}}$ the dominant term is $e^{\tilde{q}_{1}+\tilde{q}_{2}}$. Thus, one obtains for $t \rightarrow+\infty$ the limits $e^{-q_{1}} \rightarrow\left[\tilde{p}_{1}\left(\tilde{p}_{1}-\tilde{p}_{2}\right)\right]^{-1} e^{\tilde{q}_{1}}, e^{-\tilde{q}_{2}} \rightarrow\left(\tilde{p}_{1} \tilde{p}_{2}\right)^{-1} e^{\tilde{q}_{1}+\tilde{q}_{2}}$, and then $p_{1} \rightarrow-\tilde{p}_{1}+\tilde{p}_{2}$, $p_{2} \rightarrow-\tilde{p}_{2}$. With respect to the $t \rightarrow-\infty$ asymptotic momenta, the $t \rightarrow+\infty$ limits differ precisely by an application of the Weyl group element $Z=R M$ (4.1.11). Thus, as we found earlier in the Liouville case, the classical asymptotic interacting-theory momenta at $t \rightarrow \pm \infty$ differ by a Weyl-group transformation of the free momenta. Thus, in the Toda theory as well in the Liouville on, asymptotic values of interacting-theory momenta are conserved modulo the Weyl group. 


\subsection{Quantum Toda Mechanics}

The quantum generalisation of the canonical transformation (4.1.3) once again maps the interacting-theory Hamiltonian (4.3) into the free-theory Hamiltonian (4.1.4), but this time care needs to be taken of quantum operator orderings. We find that the following sequence of elementary canonical transformations implements the free-field map:

T6] $\mathcal{P}_{\left(q_{1}-q_{2}+\frac{1}{q_{1} q_{2}}, \frac{1}{q_{1}}-\frac{1}{q_{2}}+q_{1} q_{2}\right)}:\left\{\begin{array}{l}q_{1} \mapsto q_{1}^{\prime}=q_{1}-q_{2}+\frac{1}{q_{1} q_{2}}, \quad p_{1} \mapsto p_{1}^{\prime}=\frac{1}{\operatorname{det} \frac{\partial q_{i}^{\prime}}{\partial q_{j}}\left(\frac{\partial q_{2}^{\prime}}{\partial q_{2}} p_{1}-\frac{\partial q_{2}^{\prime}}{\partial q_{1}} p_{2}\right)} \\ q_{2} \mapsto q_{2}^{\prime}=\frac{1}{q_{1}}-\frac{1}{q_{2}}+q_{1} q_{2}, \quad p_{2} \mapsto p_{2}^{\prime}=\frac{1}{\operatorname{det} \frac{\partial q_{i}^{\prime}}{\partial q_{j}}}\left(\frac{\partial q_{1}^{\prime}}{\partial q_{1}} p_{2}-\frac{\partial q_{1}^{\prime}}{\partial q_{2}} p_{1}\right)\end{array}\right.$

$$
\mathcal{P}_{\left(e^{\left.q_{1}, e^{q_{2}}\right)}\right.}: \quad \begin{cases}q_{1} \mapsto e^{q_{1}}, & p_{1} \mapsto e^{-q_{1}} p_{1} \\ q_{2} \mapsto e^{q_{2}}, & p_{2} \mapsto e^{-q_{2}} p_{2} .\end{cases}
$$

Among the transformations composing this free-field map, we have a type not yet encountered in this paper, the "similarity" transformations embodied in [T1,T3,T4] (although, strictly speaking, the inverse-momentum transformations [L3] are also of this type). The coordinate similarity transformations [T3,T4], of the form $\left(p_{i} \mapsto p_{i}-f_{, i}\left(q_{j}\right), q_{i} \mapsto q_{i}\right)$ and are generated by $e^{\mathrm{i} f\left(q_{j}\right)}$, transforming wavefunctions $\Psi\left(q_{j}\right)$ into $e^{\mathrm{i} f\left(q_{j}\right)} \Psi\left(q_{j}\right)$ [19,21]. Momentum versions such as [T1], of the form $\left(q_{i} \mapsto q_{i}+f_{, i}\left(p_{j}\right), p_{i} \mapsto p_{i}\right)$, are generated by $e^{\mathrm{i} f\left(p_{j}\right)}=\mathcal{I} e^{\mathrm{i} f\left(q_{j}\right)} \mathcal{I}^{-1}$. The Gamma function in the generator of [T1] may be given an integral representation

$$
\Gamma\left(1-\mathrm{i} p_{1}-\mathrm{i} p_{2}\right)=\int_{0}^{\infty} d u u^{-\mathrm{i} p_{1}-\mathrm{i} p_{2}} e^{-u},
$$


in which the momentum operators in the exponent simply generate translations:

$$
u^{-\mathrm{i} p_{1}-\mathrm{i} p_{2}}=\exp \left(-\mathrm{i}\left(p_{1}+p_{2}\right) \ln u\right)
$$

Following the mapping of the Toda Hamiltonian (4.3) as the quantum canonical transformation [T1 - T7] unfolds, we have the sequence of stages

$$
\begin{aligned}
3 H_{\mathrm{T}} & =p_{1}^{2}+p_{2}^{2}+p_{1} p_{2}+3 e^{2 q_{1}-q_{2}}+3 e^{2 q_{2}-q_{1}} \\
& \mapsto p_{1}^{2}+p_{2}^{2}+p_{1} p_{2}-3\left(e^{2 q_{1}-q_{2}}+e^{2 q_{2}-q_{1}}\right)\left(p_{1}+p_{2}\right) \\
& \mapsto\left(q_{1} p_{1}\right)^{2}+\left(q_{2} p_{2}\right)^{2}+q_{1} p_{1} q_{2} p_{2}-3\left(\frac{q_{1}^{2}}{q_{2}}+\frac{q_{2}^{2}}{q_{1}}\right)\left(q_{1} p_{1}+q_{2} p_{2}\right) \\
& \mapsto\left(p_{1} q_{1}\right)^{2}+\left(p_{2} q_{2}\right)^{2}+p_{1} q_{1} p_{2} q_{2}-3\left(\frac{q_{1}^{2}}{q_{2}}+\frac{q_{2}^{2}}{q_{1}}\right)\left(p_{1} q_{1}+p_{2} q_{2}\right) \\
& \mapsto\left(p_{1} q_{1}\right)^{2}+\left(p_{2} q_{2}\right)^{2}+p_{1} q_{1} p_{2} q_{2}-9 q_{1} q_{2}-3 p_{2} q_{1}^{2}-3 p_{1} q_{2}^{2} \\
& \mapsto\left(q_{1} p_{1}\right)^{2}+\left(q_{2} p_{2}\right)^{2}+q_{1} p_{1} q_{2} p_{2}-9 p_{1} p_{2}+3 q_{2} p_{1}^{2}+3 q_{1} p_{2}^{2} \\
& \mapsto\left(q_{1} p_{1}\right)^{2}+\left(q_{2} p_{2}\right)^{2}-q_{1} p_{1} q_{2} p_{2} \\
& \mapsto p_{1}^{2}+p_{2}^{2}-p_{1} p_{2}=3 \tilde{H}_{\mathrm{T}} .
\end{aligned}
$$

The mapping from free-field wavefunctions to interacting Toda wavefunctions is then given by the inverse transformation generator

$$
\begin{aligned}
& C^{-1}=e^{-\frac{\pi}{2}\left(p_{1}+p_{2}\right)} \Gamma\left(1-\mathrm{i} p_{1}-\mathrm{i} p_{2}\right) \mathcal{P}_{\left(e^{\left.q_{1}, e^{q_{2}}\right)}\right.} q_{1} q_{2} \exp \left(\mathrm{i}\left(\frac{q_{1}^{2}}{q_{2}}+\frac{q_{2}^{2}}{q_{1}}\right)\right) \\
&\left.\mathcal{I}_{1}^{-1} \mathcal{I}_{2}^{-1} \mathcal{P}_{\left(q_{1}-q_{2}+\frac{1}{q_{1} q_{2}}\right.}^{-1}, \frac{1}{q_{1}}-\frac{1}{q_{2}}+q_{1} q_{2}\right) \\
& \mathcal{P}_{\left(\ln q_{1}, \ln q_{2}\right)}
\end{aligned}
$$

so that

$$
\Psi_{k_{1}, k_{2}}\left(q_{1}, q_{2}\right)=N_{k_{1} k_{2}} C^{-1} e^{i k_{1} q_{1}+i k_{2} q_{2}}
$$

where $N_{k_{1} k_{2}}$ is a normalization constant. To obtain a well-defined integral representation of the Toda wavefunction, the contours and ranges of integration for the Fourier transforms must be specified. These will be chosen so as to give a wavefunction with the physically correct asymptotic behaviour. In order to have a normalizable Toda wavefunction, the wavefunction must vanish asymptotically in the region under the potential, i.e. in the region where $2 q_{1}-q_{2}>0,2 q_{2}-q_{1}>0$.

To evaluate the wavefunction, focus first on the portion of the transformation (4.2.4) from the initial point transformation to the inverse Fourier transforms, i.e.

$$
\mathcal{I}_{1}^{-1} \mathcal{I}_{2}^{-1} \mathcal{P}_{\left(q_{1}-q_{2}+\frac{1}{q_{1} q_{2}}, \frac{1}{q_{1}}-\frac{1}{q_{2}}+q_{1} q_{2}\right)}^{-1} \mathcal{P}_{\left(\ln q_{1}, \ln q_{2}\right)} e^{\mathrm{i} k_{1} q_{1}+\mathrm{i} k_{2} q_{2}}
$$


Formally, this is

$$
\frac{1}{2 \pi} \int d z_{1} \int d z_{2} e^{-\mathrm{i} q_{1} z_{1}} e^{-\mathrm{i} q_{2} z_{2}} \mathcal{P}_{\left(y_{1}-y_{2}+\frac{1}{y_{1} y_{2}}, \frac{1}{y_{1}}-\frac{1}{y_{2}}+y_{1} y_{2}\right)}^{-1} y_{1}^{\mathrm{i} k_{1}} y_{2}^{\mathrm{i} k_{2}} .
$$

We shall determine the region of integration shortly. The inverse point transformation [T6 $]^{-1}$ implements the change of variables $z_{1}=y_{1}-y_{2}+\frac{1}{y_{1} y_{2}}, z_{2}=\frac{1}{y_{1}}-\frac{1}{y_{2}}+y_{1} y_{2}$; consequently, one obtains the integral

$$
\frac{1}{2 \pi} \int d y_{1} \int d y_{2}[\mathrm{jac}] y_{1}^{\mathrm{i} k_{1}} y_{2}^{\mathrm{i} k_{2}} e^{-\mathrm{i} q_{1}\left(y_{1}-y_{2}+\frac{1}{y_{1} y_{2}}\right)} e^{-\mathrm{i} q_{2}\left(\frac{1}{y_{1}}-\frac{1}{y_{2}}+y_{1} y_{2}\right)}
$$

where

$$
[\mathrm{jac}]=\frac{-1}{y_{1} y_{2}}\left(y_{1}+y_{2}\right)\left(-y_{2}+\frac{1}{y_{1}^{2}}\right)\left(y_{1}+\frac{1}{y_{2}^{2}}\right) .
$$

Applying the remaining transformations subsequent to the inverse Fourier transforms $[\mathrm{T} 5]^{-1}$ in (4.2.4), and acting with the translation operator inside the Gamma function, the Toda wavefunction is found to be

$$
\begin{aligned}
\Psi_{k_{1}, k_{2}}\left(q_{1}, q_{2}\right)= & \frac{N_{k_{1} k_{2}}}{2 \pi} e^{\mathrm{i} \pi} \int_{0}^{\infty} d u e^{q_{1}+q_{2}} u^{-2} e^{-u-\left(e^{2 q_{1}-q_{2}}+e^{2 q_{2}-q_{1}}\right) u^{-1}} \times \\
& \int d y_{1} \int d y_{2}[\mathrm{jac}] y_{1}^{\mathrm{i} k_{1}} y_{2}^{\mathrm{i} k_{2}} e^{e^{q_{1}}\left(y_{1}-y_{2}+\frac{1}{y_{1} y_{2}}\right) u^{-1}} e^{e^{q_{2}}\left(\frac{1}{y_{1}}-\frac{1}{y_{2}}+y_{1} y_{2}\right) u^{-1}} .
\end{aligned}
$$

The $y_{1}, y_{2}$ integrals are convergent if the ranges of integration are taken to run from $-\infty$ to 0 and from 0 to $\infty$, respectively. The $u$ integral is then seen to be convergent. These ranges are also the ones that give vanishing boundary contributions when integrating by parts in verifying that the generalised inverse Fourier transforms $\mathcal{I}_{1}^{-1} \mathcal{I}_{2}^{-1}$ correspond correctly to the inverse of the operator transformations [T5], analogously to our discussion in section two for the Liouville case.

In order to make the convergence of the integrals more explicit, we redefine $y_{1} \mapsto-y_{1}$, obtaining the manifestly convergent expression

$$
\begin{aligned}
\Psi_{k_{1}, k_{2}}\left(q_{1}, q_{2}\right)= & \frac{N_{k_{1} k_{2}}}{2 \pi} e^{\pi k_{1}} \int_{0}^{\infty} d u e^{q_{1}+q_{2}} u^{-2} e^{-u-\left(e^{2 q_{1}-q_{2}}+e^{2 q_{2}-q_{1}}\right) u^{-1}} \times \\
& \int_{0}^{\infty} d y_{1} \int_{0}^{\infty} d y_{2}[\mathrm{jac}]^{\prime} y_{1}^{\mathrm{i} k_{1}} y_{2}^{\mathrm{i} k_{2}} e^{-e^{q_{1}}\left(y_{1}+y_{2}+\frac{1}{y_{1} y_{2}}\right) u^{-1}} e^{-e^{q_{2}}\left(\frac{1}{y_{1}}+\frac{1}{y_{2}}+y_{1} y_{2}\right) u^{-1}},
\end{aligned}
$$

where

$$
[\mathrm{jac}]^{\prime}=\frac{1}{y_{1} y_{2}}\left(y_{1}-y_{2}\right)\left(y_{2}-\frac{1}{y_{1}^{2}}\right)\left(y_{1}-\frac{1}{y_{2}^{2}}\right) .
$$

The wavefunction is now seen to vanish strongly under the potential owing to the presence of the factor $\exp \left(-\left(e^{2 q_{1}-q_{2}}+e^{2 q_{2}-q_{1}}\right) u^{-1}\right)$. 
The Weyl-group symmetry of the wavefunction may now be seen directly in (4.2.11) by changing the variables of integration, again analogously to our earlier Liouville discussion. The $M$ transformation of the Weyl group corresponds to the order-three change of variables

$$
\begin{array}{ccccc}
y_{1} & \stackrel{M}{\longrightarrow} & y_{2} & \stackrel{M}{\longrightarrow} & \frac{1}{y_{1} y_{2}} \\
y_{2} & \stackrel{M}{\longrightarrow} & \frac{1}{y_{1} y_{2}} & \stackrel{M}{\longrightarrow} & y_{1}
\end{array} \quad \Leftrightarrow \quad \begin{array}{cccccc}
k_{1} & \stackrel{M}{\longrightarrow} & -k_{2} & \stackrel{M}{\longrightarrow} & k_{2}-k_{1}, \\
k_{2} & \stackrel{M}{\longrightarrow} & k_{1}-k_{2} & \stackrel{M}{\longrightarrow} & -k_{1}
\end{array},
$$

while the $R$ transformation corresponds to the order-two change of variables

$$
\begin{array}{ll}
y_{1} & \stackrel{R}{\longrightarrow} \\
y_{2} & y_{2} \\
\stackrel{R}{\longrightarrow} & y_{1}
\end{array} \quad \Leftrightarrow \quad \begin{array}{llll}
k_{1} & \stackrel{R}{\longrightarrow} & k_{2} \\
k_{2} & \stackrel{R}{\longrightarrow} & k_{1}
\end{array} .
$$

Thus, the only changes to the wavefunction (4.2.11) that arise upon making a Weyl-group transformation are the inessential ones occurring in the prefactor $e^{\pi k_{1}}$; even these will in the end be cancelled after normalization by compensating changes in $N_{k_{1}, k_{2}}$, as we saw in the Liouville case (2.2.9). Thus, the quantum Toda theory possesses the full Weyl-group symmetry that we found for Toda theory at the classical level.

\section{Conclusion}

In this paper, we have made explicit constructions of the wavefunctions of Liouville and Toda gravities, using the technique of intertwining operators to realise canonical transformations between the free and interacting theories. These detailed results show distinctly the very important rôle played by the Weyl group of the underlying $A_{N}$ Lie algebra in each case. At the classical level, this symmetry is seen through the presence of a multiplet of forms of the free-field canonical transformation map, each form being physically appropriate for a different domain in the space of free-field momenta. At the quantum level, the canonical transformations of the fundamental conjugate operator pairs $\left(p_{i}, q_{i}\right)$ must be constructed with careful attention to operator ordering.

In analysing the Weyl-group symmetries of the free-field maps, it emerges in both the Liouville and Toda cases that the most Weyl-symmetric form of the quantum canonical transformation does not itself have a direct limit to a sensible classical transformation. We saw this in the complex character of the manifestly Weyl-symmetric transformation (2.2.15) for the operator pair $(p, q)$ in the Liouville case. The Weyl-symmetric intertwining-operator map (4.2.11) in the Toda case gives rise to a similarly manifestly symmetric transformation of the operator pairs $\left(p_{i}, q_{i}\right)$, and, once again, the classical limit of this transformation can be seen to admit no real classical solutions. The manifestly Weyl-symmetric forms of these quantum transformations also generate the most manifestly-convergent forms of the quantum wavefunctions. Moreover, these manifestly-symmetric forms are also the ones that manifestly satisfy the physically-required boundary condition of falling away to zero in the region under 
the Liouville/Toda potentials. It appears that, in requiring manifest Weyl symmetry, as well as in imposing the physical boundary conditions, one is focusing on behaviour in the region under the potential, where motion is classically excluded. In both the Liouville and Toda cases, there exist other forms of the quantum transformations, obtained by analytic continuation, that do limit to classically-sensible forms [L1-L4, T1-T7]. In these classicallysensible forms of the quantum transformations, the Weyl symmetry is harder to spot, and also the integral representations for the wavefunctions lie just at the limits of convergence and hence need to be regularized. Such behaviour is of course familiar from general comparisons between Euclidean and Minkowskian approaches to quantum theory, but the involvement of the Weyl-group symmetry in the present cases is striking.

Another striking result of our investigations is the precise nature of the operator-state map, which figures in many discussions of non-critical string theory. It is important to emphasise that the operator-state map is quite distinct from the true canonical transformation between free and interacting fields that we have derived using intertwining operators. Some aspects of the operator-state map, such as the occurrence of Seiberg bounds, are specific properties of this map and do not particularly reveal features of the underlying dynamics. Use of the operator-state map is motivated by the success of conformal field theory in formulating free-field string theory in terms of vertex operators. It is not clear to us how successfully the vertex-operator approach can be carried over to the interacting Liouville/Toda cases. From the results of section three, we have seen explicitly that in the important case of imaginary free-field momentum which arises in non-critical string theories, the operator-state map does not generally produce single interacting-theory eigenfunctions. The ramifications of this observation need further study.

Although Liouville and Toda gravities have already been studied in considerable detail, the literature reveals different approaches to the subject that have not been fully crossfertilised. The above considerations of the operator-state map might be cited as an example of this. One would like to understand better how the background charges needed for conformalanomaly cancellation affect the canonical transformation to free fields. A standard approach in much of the literature is to use the operator-state map to implement a shift of the vertexoperator momenta as in (2.3). This is unproblematic in free-field string theories, but, as we have noted above, the operator-state map does not always generate single interacting-theory eigenstates. Implementation of the canonical-transformations to free fields in the Liouville or Toda theories after first using the operator-state map to shift away the background charges is not an obviously consistent procedure. On the other hand, the proper way to incorporate directly the effects of the background charges into the free-field canonical transformations is not clear.

Another sense in which the results of the different approaches to non-critical string theory need to be interrelated concerns the implications of recent results on the spectra of lowdimensional non-critical strings obtained via BRST analysis [26,27]. At the minisuperspace 
level that has been our main concern in this paper, there are no surprises. But when one includes the oscillator excitations, non-critical string theories develop significant new features not generally taken into account in Liouville or Toda field-theory discussions. In the analysis of the BRST cohomology problem to determine the spectra of ordinary strings with one or two scalars [26,27], or of $W_{3}$ strings with two scalars [36,37], it turns out that the physical states do not occur only in the ghost-vacuum sector. Indeed, it is necessary to incorporate the full set of cohomologically-nontrivial states in order to see the Weyl-group symmetry that has played a central rôle in the analysis of the present paper.

To make the rôle of ghost excitations in the excited states more clear, consider the known free-field spectrum of the two-scalar $W_{3}$ string, which corresponds, in the interacting theory, to the case of pure Toda $W_{3}$ gravity as considered in section four. The physical states are, by definition, states that are annihilated by the BRST operator, but that are not BRST trivial. Since we have only two scalar matter fields, and since there are two constraints (from the spin-2 current and the spin-3 current), it follows that there are no continuous degrees of freedom (i.e. there are no transverse spacetime dimensions), so all physical states have discrete momenta. The physical states in this case can be subdivided into two categories. First, consider the physical states that involve no excitations of the ghost fields; these are generally built by acting with vertex operators and matter-field excitations on the ghost vacuum. Since there are no transverse dimensions in the two-scalar string, however, there cannot be any such BRST non-trivial states involving matter-field excitations. Thus, the only physical states of this "standard ghost structure" are the tachyons, built by acting with matter vertex operators $e^{\alpha_{1} \varphi_{1}+\alpha_{2} \varphi_{2}}$ on the ghost vacuum. The momenta of these, and indeed of all physical states in the two-scalar $W_{3}$ string are quantized and take the form

$$
\alpha_{1}=\frac{1}{7} Q_{1} k_{1}, \quad \alpha_{2}=\frac{1}{7} Q_{2} k_{2},
$$

where $k_{1}$ and $k_{2}$ are integers. There are six tachyonic physical states, with momenta given by $(5.1)$ with $\left(k_{1}, k_{2}\right)=(-6,-6),(-6,-8),(-8,-6),(-8,-8),(-7,-5),(-7,-9)[14,38]$. These are mapped into one another under the action of the six-element Weyl group of $A_{2} \equiv$ $S U(3)$. The Weyl group acts by reflection in the momentum plane, and is most appropriately described in terms of its action on the shifted momenta $\hat{\alpha}_{i}=\alpha_{i}+Q_{i}$. Correspondingly, we may define the shifted integers $\left(\hat{k}_{1}, \hat{k}_{2}\right)=\left(k_{1}+7, k_{2}+7\right)$. The Toda momenta that we have used earlier in this article are related to the $\hat{k}_{i}$ by

$$
\begin{aligned}
\mathrm{i} p_{1} & =-\frac{1}{2} \hat{k}_{1}+\frac{1}{6} \hat{k}_{2} \\
\mathrm{i} p_{2} & =-\frac{1}{3} \hat{k}_{2} .
\end{aligned}
$$

The Weyl group is generated by the two transformations $R$ and $M$ discussed in section four, which map $\left(\hat{k}_{1}, \hat{k}_{2}\right)$ according to

$$
\begin{array}{cl}
R: & \left(\hat{k}_{1}, \hat{k}_{2}\right) \longrightarrow\left(-\frac{1}{2}\left(\hat{k}_{1}+\hat{k}_{2}\right),-\frac{1}{2}\left(3 \hat{k}_{1}-\hat{k}_{2}\right)\right) \\
M: & \left(\hat{k}_{1}, \hat{k}_{2}\right) \longrightarrow\left(-\frac{1}{2}\left(\hat{k}_{1}-\hat{k}_{2}\right),-\frac{1}{2}\left(3 \hat{k}_{1}+\hat{k}_{2}\right)\right) .
\end{array}
$$


The six elements of the Weyl group are given by $1, R, M, R M, M^{2}$ and $R M^{2}$. One can easily verify that the six tachyon momenta given above are mapped into one another under these transformations. ${ }^{\dagger}$

The above six tachyons are the only physical states without ghost excitations in the two-scalar $W_{3}$ string. Thus, as it stands, the minisuperspace discussion of Toda quantum mechanics given in section four is really only applicable as a tachyon-sector approximation to Toda field theory in the context of pure $W_{3}$ gravity. The two-scalar $W_{3}$ string has infinitely many more physical states, but all except the tachyons have "non-standard" ghost structure, involving excitations of the ghosts as well as of the matter fields. Thus, they fall outside the context of our section-four discussion. Ultimately, one would like to be able to extend the discussion of Toda field theory to include the full spectrum of physical states in $W_{3}$ gravity, including those of non-standard ghost structure. To our knowledge, the analogous issues have not yet been addressed in the literature even for Liouville gravity. In Liouville theory, the majority of physical states for the pure gravity case (i.e. for the one-scalar string) also have non-standard ghost structure. Thus, one can expect that a full treatment of Liouville or Toda field theory should include the ghosts as well as the matter fields.

The higher-level physical states of the free-field two-scalar $W_{3}$ string also fall into multiplets under the Weyl group of $A_{2}$. The states in a multiplet all have the same level number $\ell$, but, unlike the case of the tachyons at level $\ell=0$, the various members of an $\ell \neq 0$ multiplet have different ghost numbers. Further details may be found in [37], but the essential structure is as follows. A necessary condition that must be satisfied by any physical state is that it be described by an operator with conformal dimension zero: this is the mass-shell condition. For a state at level $\ell$, this implies that the shifted integers describing the momentum satisfy

$$
3 \hat{k}_{1}^{2}+\hat{k}_{2}^{2}=4(12 \ell+1) .
$$

Remarkably, it turns out [37] that any solution of (5.5) for integers $\hat{k}_{1}, \hat{k}_{2}$ and $\ell$ gives momenta corresponding to some physical state of the theory. Now, it is easy to see that the mass-shell condition (5.5) is preserved by the Weyl group generated by $R$ and $M$ as given in (5.3, 5.4). Thus, it follows that the Weyl group acts on the momentum of any physical state to give other physical-state momenta. Consequently, one can associate the physical states together into multiplets by the action of the Weyl group. In the case of the tachyons at $\ell=0$, they all have the standard ghost structure, and hence in particular have the same ghost number. For the higher-level states, which have non-standard ghost structure, the physical states with Weyl-related momenta at a given level $\ell$ do not all have the same ghost number. As we have seen, the Weyl group plays an essential rôle in relating the Toda and the free-field

$\dagger$ In comparing the transformations $(5.3,5.4)$ with our earlier forms $(4.1 .8,4.1 .9)$, it is necessary to recall the difference between the free-field limit of the interacting Toda variables $(p, q)$ and the free-field variables $(\tilde{p}, \tilde{q})$. As noted in section four, one needs to apply a basis-change transformation, e.g. $\left(p_{1}, q_{1}\right) \mapsto\left(-\tilde{p}_{1},-\tilde{q}_{1}\right)$, in order to relate the two. 
wavefunctions and operators. For the full non-critical $W_{3}$ string theory, these observations underline the importance of correctly taking the ghosts into account.

\section{ACKNOWLEDGMENTS}

We would like to thank H. Lu, M. Olshanetsky, J. Schnittger and Y. Tanii for helpful discussions. For hospitality during the course of the work, C.N.P. would like to thank Chalmers University (Gothenburg), Imperial College (London) and SISSA (Trieste); K.S.S. would like to thank Chalmers University, J.I.N.R. (Dubna), the Laboratoire de Physique Théorique de l'Ecole Normale Supérieure (Paris), SISSA and Texas A\&M University. 


\section{Appendix. Other Toda wavefunction representations}

The integral representations of the Liouville and Toda wavefunctions quoted in the review by Olshanetsky and Perelomov [28] can be economically derived using canonical transformations. Two of these were obtained in [29] by constructing power series solutions for the wavefunctions and then by simply stating the associated integral representations. A third, the result of Vinogradov and Takhtadjan [30] for the 3-body Toda wavefunction, was obtained using methods proper to number theory. It is useful to have explicit elementary derivations of these integral representations.

The key difference between these other representations and those given in the body of this paper is that they are not obtained by transforming all the way to a free theory, but rather by transforming to an interacting theory for which one particular solution can be found by inspection. The result is that one has an eigenvalue-dependent transformation which produces a particular solution. This can be a powerful method for constructing particular solutions to a theory [22].

The result of Vinogradov and Takhtadjan [30] for the 3-body Toda wavefunction is the most easily obtained and so we shall begin with it. We start from the 3-body Toda Hamiltonian

$$
H=\frac{1}{2}\left(p_{1}^{2}+p_{2}^{2}+p_{3}^{2}\right)+e^{q_{1}-q_{2}}+e^{q_{2}-q_{3}} .
$$

This can be transformed into center-of-mass coordinates in the Chevalley basis of $A_{2}$ by the transformation $\ddagger$

$$
\begin{array}{llllll}
q_{1} & \mapsto & q_{1}+\frac{1}{3} q_{3}, & p_{1} & \mapsto & \frac{2}{3} p_{1}+\frac{1}{3} p_{2}+p_{3} \\
q_{2} & \mapsto-q_{1}+q_{2}+\frac{1}{3} q_{3}, & & p_{2} & \mapsto & -\frac{1}{3} p_{1}+\frac{1}{3} p_{2}+p_{3} \\
q_{3} & \mapsto-q_{2}+\frac{1}{3} q_{3}, & & p_{3} & \mapsto & -\frac{1}{3} p_{1}-\frac{2}{3} p_{2}+p_{3} .
\end{array}
$$

The resulting Hamiltonian is then of the form used in section four, but including the centerof-mass variables,

$$
H^{a}=\frac{1}{3}\left(p_{1}^{2}+p_{2}^{2}+p_{1} p_{2}\right)+\frac{3}{2} p_{3}^{2}+e^{2 q_{1}-q_{2}}+e^{2 q_{2}-q_{1}} .
$$

The following sequence of transformations $\S$ then reduces the Hamiltonian to a form for

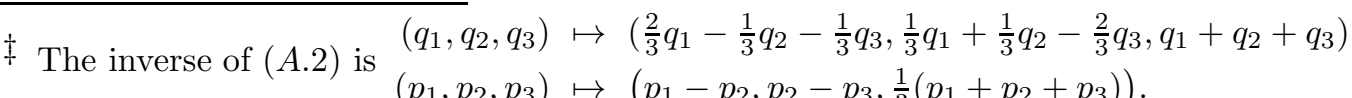

$\S$ The inverse transformation $\mathcal{P}_{\mathrm{VT} 1}^{-1}$ takes $\left(q_{1}, q_{2}\right) \mapsto\left(\frac{1}{2}\left(2 q_{1}-q_{2}\right), \frac{1}{2}\left(2 q_{2}-q_{1}\right)\right)$.
} 
which an eigenfunction can be found by inspection.
[VT1]
$\mathcal{P}_{\mathrm{VT} 1}: \quad\left\{\begin{array}{l}q_{1} \mapsto \frac{2}{3}\left(2 q_{1}+q_{2}\right), \\ q_{2} \mapsto \frac{2}{3}\left(2 q_{2}+q_{1}\right),\end{array}\right.$
$p_{1} \mapsto p_{1}-\frac{1}{2} p_{2}$
$p_{2} \mapsto p_{2}-\frac{1}{2} p_{1}$
$[\mathrm{VT} 2] \exp \left(-i \ln 2\left(p_{1}+p_{2}\right)\right):\left\{\begin{array}{l}q_{1} \mapsto q_{1}-\ln 2, \\ q_{2} \mapsto q_{2}-\ln 2,\end{array}\right.$
$p_{1} \mapsto p_{1}$
$p_{2} \mapsto p_{2}$
[VT3] $\exp \left(-i k\left(q_{1}-q_{2}\right)\right): \quad\left\{\begin{array}{l}q_{1} \mapsto q_{1}, \\ q_{2} \mapsto q_{2},\end{array}\right.$
$p_{1} \mapsto p_{1}+k$
$p_{2} \mapsto p_{2}-k$
[VT4]

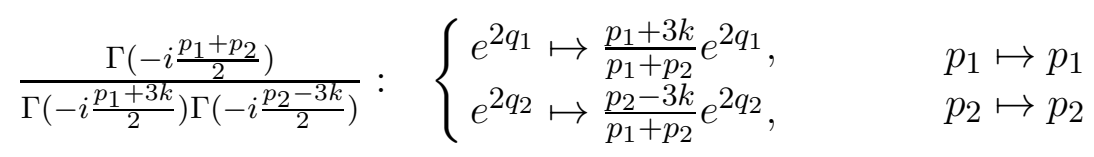

Suppressing the free-particle kinetic term $\frac{3}{2} p_{3}^{2}$ describing the motion of the center of mass, the Hamiltonian transforms as follows:

[VT1]

$[\mathrm{VT} 2]$

[VT3]

[VT4]

$$
\begin{aligned}
4 H^{a} & =\frac{4}{3}\left(p_{1}^{2}+p_{2}^{2}+p_{1} p_{2}\right)+4 e^{2 q_{1}-q_{2}}+4 e^{2 q_{2}-q_{1}} . \\
& \mapsto p_{1}^{2}+p_{2}^{2}-p_{1} p_{2}+4 e^{2 q_{1}}+4 e^{2 q_{2}} \\
& \mapsto p_{1}^{2}+p_{2}^{2}-p_{1} p_{2}+e^{2 q_{1}}+e^{2 q_{2}} \\
& \mapsto p_{1}^{2}+3 k p_{1}+p_{2}^{2}-3 k p_{2}-p_{1} p_{2}+e^{2 q_{1}}+e^{2 q_{2}}+3 k^{2} \\
& \mapsto \frac{1}{p_{1}+p_{2}}\left(\left(p_{1}+3 k\right)\left(p_{1}^{2}+e^{2 q_{1}}\right)+\left(p_{2}-3 k\right)\left(p_{2}^{2}+e^{2 q_{2}}\right)\right)+3 k^{2} .
\end{aligned}
$$

Inspection shows that this final separable Hamiltonian has $K_{\nu}\left(e^{q_{1}}\right) K_{\nu}\left(e^{q_{2}}\right)$ as an eigenfunction, with eigenvalue $\frac{1}{4}\left(-\nu^{2}+3 k^{2}\right)$. It is also an eigenfunction of the transformed $W$ operator invariant with eigenvalue $-\frac{3}{8} k\left(k^{2}+\nu^{2}\right)$.

Applying the intertwining operator, we obtain an eigenfunction of the Toda Hamiltonian (A.3) (with zero center-of-mass momentum):

$$
\begin{aligned}
& \psi_{k, \nu}\left(q_{1}, q_{2}\right)=N_{k, \nu} \mathcal{P}_{\mathrm{VT} 1}^{-1} \exp \left(i\left(p_{1}+p_{2}\right) \ln 2\right) \exp \left(i k\left(q_{1}-q_{2}\right)\right) \\
& \frac{\Gamma\left(-i \frac{p_{1}+3 k}{2}\right) \Gamma\left(-i \frac{p_{2}-3 k}{2}\right)}{\Gamma\left(-i \frac{p_{1}+p_{2}}{2}\right)} K_{\nu}\left(e^{q_{1}}\right) K_{\nu}\left(e^{q_{2}}\right) .
\end{aligned}
$$

Recognizing the product of Gamma functions as a Beta function, and using a familiar integral representation for the Beta function, one finds the result, in the Chevalley basis,

$$
\begin{aligned}
& \psi_{k, \nu} \\
& =N_{k, \nu} \mathcal{P}_{\mathrm{VT} 1}^{-1} e^{\mathrm{i} k\left(q_{1}-q_{2}\right)} \int_{0}^{\infty} d t t^{-1-\frac{1}{2} \mathrm{i}\left(p_{2}-3 k\right)}(1+t)^{\frac{1}{2} \mathrm{i}\left(p_{1}+p_{2}\right)} K_{\nu}\left(2 e^{q_{1}}\right) K_{\nu}\left(2 e^{q_{2}}\right) \\
& =N_{k, \nu} e^{\frac{3}{2} \mathrm{i} k\left(q_{1}-q_{2}\right)} \int_{0}^{\infty} d t t^{-1+\frac{3}{2} \mathrm{i} k} K_{\nu}\left(2 \sqrt{(1+t)} e^{\frac{1}{2}\left(2 q_{1}-q_{2}\right)}\right) K_{\nu}\left(2 \sqrt{\left(1+t^{-1}\right)} e^{\frac{1}{2}\left(2 q_{2}-q_{1}\right)}\right) .
\end{aligned}
$$


Upon making an asymptotic expansion, one finds that this integral corresponds to the solution (4.2.10) with $k=-\left(k_{1}+k_{2}\right) / 3, \nu=i\left(k_{1}-k_{2}\right)$. Transforming back into the coordinates of the original Hamiltonian $(A .1)$, one finds

$$
\begin{aligned}
& \Psi_{k, \nu}= N_{k, \nu} \exp \left(\frac{1}{2} \mathrm{i} k\left(q_{1}-2 q_{2}+q_{3}\right)\right) \int_{0}^{\infty} d t t^{-1+\frac{3}{2} \mathrm{i} k} \times \\
& K_{\nu}\left(2 \sqrt{(1+t)} e^{\frac{1}{2}\left(q_{1}-q_{2}\right)}\right) K_{\nu}\left(2 \sqrt{\left(1+t^{-1}\right)} e^{\frac{1}{2}\left(q_{2}-q_{3}\right)}\right) .
\end{aligned}
$$

With the identifications $k=-\frac{1}{2} \mathrm{i}(t-s), \nu=-1+\frac{1}{2}(3 s+t)$, this agrees with the result of Vinogradov and Takhtadjan, as quoted in [28].

The Liouville eigenfunctions can be found by a similar procedure. The motivating idea for this form is to factor out the asymptotic plane-wave behaviour of the Liouville wavefunction and to express the solution in terms of this plane wave. The sequence of transformations and their effect on the Hamiltonian $H=p^{2}+e^{q}$ (note the difference in the argument of the exponential from the Hamiltonian given in section two) is

$$
\begin{aligned}
& H= & p^{2}+e^{q} \\
e^{-i k q}: & & \mapsto(p+k)^{2}+e^{q} \\
\mathcal{P}_{\ln q}: & & \mapsto(q p+k)+q \\
\mathcal{I}: & & \mapsto(-p q+k)^{2}+p \\
\mathcal{P}_{1 / q}: & & \mapsto\left(q^{2} p q^{-1}+k\right)^{2}-q^{2} p \\
q^{2 i k-1}: & & \mapsto(q p-k)^{2}-q p q+2 k q=(q p-2 k) q(p-1)+k^{2} .
\end{aligned}
$$

The final Hamiltonian has $e^{i q}$ as an eigenfunction, with eigenvalue $k^{2}$. The wavefunction it produces is

$$
\begin{aligned}
\psi_{k}(q) & =N_{k} e^{\mathrm{i} k q} \mathcal{P}_{e^{q}} \mathcal{I}^{-1} \mathcal{P}_{1 / q}^{-1} q^{1-2 \mathrm{i} k} e^{\mathrm{i} q} \\
& =N_{k} e^{\mathrm{i} k q} \int_{0}^{\infty} d u u^{-1-2 i k} e^{\mathrm{i}\left(u-e^{q} u^{-1}\right)} .
\end{aligned}
$$

Rotating the contour of the $u$ integration onto the positive imaginary axis and redefining $u \mapsto u e^{\mathrm{i} \pi / 2}$, one finds the result

$$
\begin{aligned}
\psi_{k}(q) & =N_{k} e^{\pi k} e^{\mathrm{i} k q} \int_{0}^{\infty} d u u^{-1-2 \mathrm{i} k} e^{-\left(u+e^{q} / u\right)} \\
& =2 N_{k} e^{\pi k} K_{2 \mathrm{i} k}\left(2 e^{\frac{1}{2} q}\right) .
\end{aligned}
$$

The Toda solution given by Bruschi et al. [29] can similarly be found by this approach. The key idea, as in the Liouville case, is to begin by factoring out the asymptotic plane-wave 
behaviour. We shall denote the momentum of this asymptotic plane wave by $\lambda=\left(\lambda_{1}, \lambda_{2}, \lambda_{3}\right)$ in the coordinate system where the Toda Hamiltonian takes the form (A.1). We also let $a=\lambda_{1}-\lambda_{2}, b=\lambda_{2}-\lambda_{3}$. The sequence of transformations to a theory for which an eigenfunction can be recognized by inspection is

$$
\begin{aligned}
& H= \frac{1}{2}\left(p_{1}^{2}+p_{2}^{2}+p_{3}^{2}\right)+e^{q_{1}-q_{2}}+e^{q_{2}-q_{3}} \\
& e^{-\mathrm{i} \lambda \cdot q}: \quad \mapsto \frac{1}{2}\left(\left(p_{1}+\lambda_{1}\right)^{2}+\left(p_{2}+\lambda_{2}\right)^{2}+\left(p_{3}+\lambda_{3}\right)^{2}\right)+e^{q_{1}-q_{2}}+e^{q_{2}-q_{3}} \\
& \mathcal{P}_{\text {C.O.M. }: \quad \mapsto} p_{1}^{2}+p_{2}^{2}-p_{1} p_{2}+a p_{1}+b p_{2}+e^{q_{1}}+e^{q_{2}} \\
&+\frac{3}{2} p_{3}^{2}+\left(\lambda_{1}+\lambda_{2}+\lambda_{3}\right) p_{3}+\frac{1}{2}\left(\lambda_{1}^{2}+\lambda_{2}^{2}+\lambda_{3}^{2}\right) \\
& \frac{\mapsto}{\Gamma\left(-\mathrm{i}\left(b+p_{2}\right)\right) \Gamma\left(1+\mathrm{i}\left(a+p_{1}\right)\right)} \frac{1}{a+b+p_{1}+p_{2}}\left[\left(a+p_{1}\right)\left(p_{1}^{2}+(a+b) p_{1}+e^{q_{1}}\right)\right. \\
&\left.+\left(b+p_{2}\right)\left(p_{2}^{2}+(a+b) p_{2}-e^{q_{2}}\right)\right] \\
&+\frac{3}{2} p_{3}^{2}+\left(\lambda_{1}+\lambda_{2}+\lambda_{3}\right) p_{3}+\frac{1}{2}\left(\lambda_{1}^{2}+\lambda_{2}^{2}+\lambda_{3}^{2}\right) \\
& e^{\frac{1}{2} \mathrm{i}(a+b)\left(q_{1}+q_{2}\right)}: \quad \frac{1}{p_{1}+p_{2}}\left[\left(p_{1}+\frac{1}{2}(a-b)\right)\left(p_{1}^{2}+e^{q_{1}}\right)+\left(p_{2}+\frac{1}{2}(b-a)\right)\left(p_{2}^{2}-e^{q_{2}}\right)\right] \\
&+\frac{3}{2} p_{3}^{2}+\left(\lambda_{1}+\lambda_{2}+\lambda_{3}\right) p_{3}-\frac{(a+b)^{2}}{4}+\frac{1}{2}\left(\lambda_{1}^{2}+\lambda_{2}^{2}+\lambda_{3}^{2}\right)
\end{aligned}
$$

where $\mathcal{P}_{\text {C.O.M. }}:\left(q_{1}, q_{2}, q_{3}\right) \mapsto\left(\frac{1}{3}\left(2 q_{1}+q_{2}+q_{3}\right), \frac{1}{3}\left(-q_{1}+q_{2}+q_{3}\right), \frac{1}{3}\left(-q_{1}-2 q_{2}+q_{3}\right)\right)$. The final Hamiltonian in this sequence has $K_{\nu}\left(2 e^{q_{1} / 2}\right) H_{\nu}^{(1)}\left(2 e^{q_{2} / 2}\right)$ as an eigenfunction with eigenvalue $-\frac{1}{4} \nu^{2}-\frac{1}{4}(a+b)^{2}+\frac{1}{2}\left(\lambda_{1}^{2}+\lambda_{2}^{2}+\lambda_{3}^{2}\right)$. Choosing $\nu=\mathrm{i}(a+b)$ gives an eigenvalue $\frac{1}{2}\left(\lambda_{1}^{2}+\lambda_{2}^{2}+\lambda_{3}^{2}\right)$ for a state which is asymptotically free, and with asymptotic momentum $\lambda=\left(\lambda_{1}, \lambda_{2}, \lambda_{3}\right)$. This corresponds to the Toda solution of Bruschi et al. Assembling the intertwining operator, the solution is

$$
\begin{gathered}
\Psi_{\lambda}(q)=N_{\lambda} e^{\mathrm{i} \lambda \cdot q} \mathcal{P}_{\text {C.O.M. }}^{-1} \frac{\Gamma\left(-\mathrm{i}\left(b+p_{2}\right)\right) \Gamma\left(1+\mathrm{i}\left(a+b+p_{1}+p_{2}\right)\right)}{\Gamma\left(1+\mathrm{i}\left(a+p_{1}\right)\right)} e^{-\frac{1}{2} \mathrm{i}(a+b)\left(q_{1}+q_{2}\right)} \\
K_{\mathrm{i}(a+b)}\left(2 e^{\frac{1}{2} q_{1}}\right) H_{\mathrm{i}(a+b)}^{(1)}\left(2 e^{\frac{1}{2} q_{2}}\right) .
\end{gathered}
$$

The product of Gamma functions is once again a Beta function and has the integral representation (with parameters at the limits of convergence)

$$
\frac{\Gamma\left(-\mathrm{i}\left(b+p_{2}\right)\right) \Gamma\left(1+\mathrm{i}\left(a+b+p_{1}+p_{2}\right)\right)}{\Gamma\left(1+\mathrm{i}\left(a+p_{1}\right)\right)}=e^{-\left(b+p_{2}\right) \pi} \int_{0}^{\infty} d \tilde{z}_{3}\left(1-\tilde{z}_{3}\right)^{-\mathrm{i} a-1-\mathrm{i} p_{1}} \tilde{z}_{3}^{-\mathrm{i} b-1-\mathrm{i} p_{2}} .
$$

Allowing the translation operators in the Beta-function integral to act, this gives the integral representation

$$
\begin{array}{r}
\Psi_{\lambda}(q)=\frac{-2 \mathrm{i} N_{\lambda}}{\pi} e^{\mathrm{i} \lambda \cdot q} e^{-b \pi} \int_{0}^{\infty} d \tilde{z}_{3}\left(1-\tilde{z}_{3}\right)^{-\mathrm{i} a-1} \tilde{z}_{3}^{-\mathrm{i} b-1} e^{-\frac{1}{2} \mathrm{i}(a+b)\left(q_{1}-q_{3}\right)} \\
\left.K_{\mathrm{i}(a+b)}\left(\frac{2}{\sqrt{1-\tilde{z}_{3}}} e^{\frac{1}{2}\left(q_{1}-q_{2}\right)}\right) K_{\mathrm{i}(a+b)}\left(\frac{2}{\sqrt{\tilde{z}_{3}}} e^{\frac{1}{2}\left(q_{2}-q_{3}\right)}\right)\right) .
\end{array}
$$


The integral representation implied by $(A .7, A .8)$ can be used for the Bessel functions here to give

$$
\begin{aligned}
\Psi_{\lambda}(q)= & \frac{-\mathrm{i} N_{\lambda}}{2 \pi} e^{-\pi(a+2 b)} e^{\mathrm{i} \lambda \cdot q} \int_{0}^{\infty} d \tilde{z}_{3}\left(1-\tilde{z}_{3}\right)^{-\mathrm{i} a-1} \tilde{z}_{3}^{-\mathrm{i} b-1} \\
& \int_{0}^{\infty} d z_{1} \int_{0}^{\infty} d z_{2}\left(z_{1} z_{2}\right)^{-\mathrm{i}(a+b)-1} e^{\mathrm{i}\left(z_{1}+z_{2}\right)} \exp \left(-\mathrm{i} \frac{e^{q_{1}-q_{2}}}{z_{2}\left(1-\tilde{z}_{3}\right)}-\mathrm{i} \frac{e^{q_{2}-q_{3}}}{z_{1} \tilde{z}_{3}}\right) .
\end{aligned}
$$

Finally, absorbing the constant factors into the normalization and making the change of variables $\tilde{z}_{3}=z_{3} /\left(z_{1} z_{2}\right)$, one reaches the form of Bruschi et al.

$$
\begin{aligned}
\Psi_{\lambda}(q)=N_{\lambda}^{\prime} e^{\mathrm{i} \lambda \cdot q} \int_{0}^{\infty} \int_{0}^{\infty} \int_{0}^{\infty} d z_{1} d z_{2} d z_{3}\left(z_{1} z_{2}-z_{3}\right)^{\mathrm{i}\left(\lambda_{2}-\lambda_{1}\right)-1} z_{3}^{\mathrm{i}\left(\lambda_{3}-\lambda_{2}\right)-1} \\
e^{\mathrm{i}\left(z_{1}+z_{2}\right)} \exp \left(-\mathrm{i} \frac{z_{1}}{z_{1} z_{2}-z_{3}} e^{q_{1}-q_{2}}-\mathrm{i} \frac{z_{2}}{z_{3}} e^{q_{2}-q_{3}}\right) .
\end{aligned}
$$

Note that this corrects some typos in the formula quoted in [28], most importantly concerning the range of integration. Note also that this is an eigenfunction of $(A .1)$ and not of the Hamiltonian of the form discussed in Section 12 of [28].

\section{REFERENCES}

[1] A.M. Polyakov, Phys. Lett. B103 (1981) 207, 211.

[2] E. D'Hoker and D.H. Phong, Rev. Mod. Phys. 60 (1988) 917;

E. D'Hoker, "Lecture notes on 2-D quantum gravity and Liouville theory,"

[3] L. Alvarez-Gaumé, "Topics in Liouville theory," in the proceedings of the Trieste Spring School, 1991 (World Scientific, 1992).

[4] N. Seiberg, "Notes on quantum Liouville theory and quantum gravity," in Common trends in mathematics and quantum field theory, Proc. of the 1990 Yukawa International Seminar, Prog. Theor. Phys. Suppl. 102 (1991).

[5] P. Ginsparg and G. Moore, "Lectures on 2D gravity and 2D string theory," presented at the TASI Summer School, Boulder, 1992, hep-th/9304011.

[6] D. Friedan, "Introduction to Polyakov's string model," in Recent Advances in Field Theory and Statistical Physics, eds J.-B. Zuber and R. Stora, (World Scientific, Singapore, 1986);

O. Alvarez, in Unified String Theory, eds M. Green and D. Gross (World Scientific, 1986).

[7] J.-L. Gervais and A. Neveu, Nucl. Phys. B199 (1982) 59; B209 (1982) 125; B224 (1983) 329; B238 (1984) 125; B238 (1984) 396; B257 [FS14] (1985) 59; B264 (1986) 557; Commun. Math. Phys. 100 (1985) 15; Phys. Lett. B151 (1985) 271.

[8] J.-L. Gervais, Commun. Math. Phys. 130 (1990) 257; 138 (1991) 301; Phys. Lett. B243 (1990) 85; Nucl. Phys. B391 (1993) 287. 
[9] J.-L. Gervais and J. Schnittger, "The many faces of the quantum Liouville exponentials," LPTENS-93/30, hep-th/9308134.

[10] H.J. Otto and G. Weigt, Phys. Lett. B159 (1985) 341; Z. Phys. C31 (1986) 219; G. Weigt, Phys. Lett. B277 (1992) 79; "Canonical quantization of the Liouville theory, quantum group structures and correlation functions," in Pathways to fundamental theories, Proc. Johns Hopkins Workshop on Current Problems in Particle Theory 16 (World Scientific, 1993).

[11] T.L. Curtright and C.B. Thorn, Phys. Rev. Lett. 48 (1982) 1309;

E. Braaten, T.L. Curtright and C.B. Thorn, Phys. Lett. B118 (1982) 115; Ann. Phys. 147 (1983) 365;

E. Braaten, T.L. Curtright, G. Ghandour and C.B. Thorn, Phys. Rev. Lett. 51 (1983) 19; Ann. Phys. 153 (1984) 147.

[12] Y. Kazama and H. Nicolai, "On the exact operator formalism of two-dimensional Liouville quantum gravity in Minkowski spacetime," DESY 93-043, UT Komaba 93-6, hep-th/9305023.

[13] A.B. Zamolodchikov, Teor. Mat. Fiz. 65 (1985) 1205.

[14] S.R. Das, A. Dhar and S.K. Rama, Mod. Phys. Lett. A6 (1991) 3055; Int. J. Mod. Phys. A7 (1992) 2295.

[15] J. Thierry-Mieg, Phys. Lett. B197 (1987) 368.

[16] L.J. Romans, Nucl. Phys. B352 (1991) 829.

[17] M. Bershadsky, W. Lerche, D. Nemeschansky and N.P. Warner, Phys. Lett. B292 (1992) 35; Nucl. Phys. B401 (1993) 304.

[18] M. Toda, Phys. Rep. 18 (1975) 1.

[19] A. Anderson and R. Camporesi, Commun. Math. Phys. 130 (1990) 61;

A. Anderson, Phys. Lett. B319 (1993) 157.

[20] A. Anderson, Phys. Rev. D47 (1993) 4458.

[21] A. Anderson, "Canonical transformations in quantum mechanics," Imperial/TP/9293/31, hep-th/9305054.

[22] A. Anderson, "Special functions from quantum canonical transformations," Imperial/TP/93-94/5, hep-th/9310168.

[23] P. Mansfield, Nucl. Phys. B208 (1982) 277.

[24] D.I. Olive, "Lectures on gauge theories and Lie algebras with some applications to spontaneous symmetry breaking and integrable dynamical systems," lectures given at University of Virginia, Charlottesville, Imperial College preprint, 1982.

[25] E. D'Hoker and R. Jackiw, Phys. Rev. D26 (1982) 3517;

A. Kihlberg, Phys. Rev. D27 (1983) 2542;

R. Marnelius, Nucl. Phys. B261 (1985) 319.

[26] B.H. Lian and G.J. Zuckerman, Phys. Lett. 254B (1991) 417; Phys. Lett. 266B (1991) 21; Commun. Math. Phys. 145 (1992) 561.

[27] E. Witten, Nucl. Phys. B373 (1992) 187;

E. Witten and B. Zwiebach, Nucl. Phys. B377 (1992) 55.

[28] M.A. Olshanetsky and A.M. Perelomov, Phys. Rep. 94 (1983) 313. 
[29] M. Bruschi, D. Levi, M.A. Olshanetsky, A.M. Perelomov and O. Ragnisco, Phys. Lett. A88 (1982) 7.

[30] A.I. Vinogradov and A.A. Takhtadjan, "Theory of the Eisenstein series for the group $S L(3, \mathbb{R})$ and its application to a binary problem, I," Notes at the LOMI seminars $\mathbf{7 6}$ (1978) 5.

[31] J. Polchinski, "Remarks on the Liouville field theory," in Strings '90, eds R. Arnowitt, R. Bryan, M.J. Duff, D. Nanopoulos, C.N. Pope and E. Sezgin (World Scientific, 1991).

[32] L. Johansson and R. Marnelius, Nucl. Phys. B254 (1985) 201; C.R. Preitschopf and C.B. Thorn, Phys. Lett. B250 (1990) 79.

[33] T. Curtright, "Quantum Bäcklund transformations and conformal algebras," in Differential Geometric Methods in Theoretical Physics, eds L.L. Chau and W. Nahm (Plenum Press, NY, 1990).

[34] G.N. Watson, A treatise on the theory of Bessel functions, 2'nd edition, (Cambridge University Press, 1944).

[35] H. Flaschka, Phys. Rev. B9 (1974) 1924.

[36] S.K. Rama, Mod. Phys. Lett. A6 (1991) 3531;

C.N. Pope, E. Sezgin, K.S. Stelle and X.J. Wang, Phys. Lett. B299 (1993) 247;

H. Lu, C.N. Pope, S. Schrans and X.J. Wang, Nucl. Phys. B408 (1993) 3;

P. Bouwknegt, J. McCarthy and K. Pilch, Commun. Math. Phys. 145 (1992) 541.

[37] H. Lu, C.N. Pope, X.J. Wang and K.W. Xu, "The complete cohomology of the $W_{3}$ string," preprint CTP TAMU-50/93, hep-th/9309041, to appear in Class. Quantum Grav.

[38] C.N. Pope, L.J. Romans and K.S. Stelle, Phys. lett. B268 (1991) 167; Phys. lett. B269 (1991) 287 ;

C.N. Pope, L.J. Romans, E. Sezgin and K.S. Stelle, Phys. Lett. 274B (1992) 298;

H. Lu, B.E.W. Nilsson, C.N. Pope, K.S. Stelle and P.C. West, Int. J. Mod. Phys. A8 (1993) 4071. 